\title{
Smooth linearization of planar periodic maps
}

\author{
A. Cima*, A. Gasull*, F. Mañosas* and R. Ortega* \\ *Departament de Matemàtiques \\ Universitat Autònoma de Barcelona, Barcelona, Spain \\ \{cima, gasull, manyosas\}@mat .uab. cat \\ *Departamento de Matemática Aplicada, \\ Universidad de Granada, Granada, Spain \\ rortega@ugr.es
}

\begin{abstract}
The celebrated Kerékjártó theorem asserts that planar continuous periodic maps can be continuously linearized. We prove that for each $k \in\{1,2, \ldots, \infty\}, \mathcal{C}^{k}$-planar periodic maps can be $\mathcal{C}^{k}$-linearized. We apply this extension to obtain a Floquet type result for some non-autonomous planar periodic ordinary differential equations and also to characterize stable fixed points of smooth planar area preserving maps that reverse orientation.
\end{abstract}

2000 MSC: Primary: 37C15. Secondary: 37C05, 54H20.

Keywords: Kerékjártó theorem, Periodic map, Linearization, Involution, Area preserving map, Floquet theory.

\section{Introduction}

A continuous map $F: \mathbb{R}^{n} \rightarrow \mathbb{R}^{n}$ satisfying $F^{m}=\mathrm{Id}$ is called $m$-periodic. Here $F^{j}=F \circ F^{j-1}$ and $m$ is the smallest positive natural number with this property. Usually, 2-periodic maps are called involutions. The simplest examples of periodic maps are found in the class of linear maps. An endomorphism $L: \mathbb{R}^{n} \rightarrow \mathbb{R}^{n}$ is periodic if it is diagonalizable in $\mathbb{C}$ and all eigenvalues are roots of unity. It seems natural to ask if these are the only possible examples, meaning that nonlinear periodic maps are indeed equivalent to linear maps. The answer to this question depends upon the dimension $n$ and also on the type of equivalence under consideration.

To initiate the discussions we consider the notion of equivalence induced by topological conjugacy. A map $F: \mathbb{R}^{n} \rightarrow \mathbb{R}^{n}$ is said (globally) $\mathcal{C}^{0}$-linearizable if there exists a homeomorphism $\psi: \mathbb{R}^{n} \rightarrow \mathbb{R}^{n}$, such that $L=\psi \circ F \circ \psi^{-1}$ is a linear map. The couple $(L, \psi)$ is called a linearization of $F$. Notice that we have emphasized the global nature in the above definition. Although many results in the theory of dynamical systems are concerned with linearization, most of them are of local nature. This is the case for the well-known Hartman-Grossman theorem.

In dimension $n=1$ it is not hard to prove that all periodic maps are $\mathcal{C}^{0}$ - linearizable with $L(x)=x$ or $L(x)=-x$. A similar result holds for $n=2$, now $L$ is either the symmetry or a rotation of angle commensurable with $2 \pi$.

Theorem 1.1. (Kerékjártó theorem) Let $F: \mathbb{R}^{2} \rightarrow \mathbb{R}^{2}$ be a continuous m-periodic map. Then $F$ is $\mathcal{C}^{0}$-linearizable. 
This result goes back to 1919 and appeared in the works of Brouwer and Kerékjártó. Currently it is known as Kerékjártó theorem. A complete proof was presented by Eilenberg in 1934 (see [6] for more details). Later it was discovered that this theorem cannot be extended to higher dimensions. In fact, in [1, 2], Bing constructed examples showing that, for any $m \geq 2$, there are continuous $m$-periodic maps in $\mathbb{R}^{3}$ which are not linearizable.

Let us now assume that the map $F$ is smooth and consider the equivalence induced by smooth conjugacy. Given $k=1,2, \ldots, \infty$, we say that a map $F: \mathbb{R}^{n} \rightarrow \mathbb{R}^{n}$ of class $\mathcal{C}^{k}$ is (globally) $\mathcal{C}^{k}$ linearizable if it is conjugate to a linear map $L$ via a $\mathcal{C}^{k}$-diffeomorphism $\psi: \mathbb{R}^{n} \rightarrow \mathbb{R}^{n}$. In dimension $n=1$ every non-trivial $\mathcal{C}^{k}$-periodic map is an involution and can be written as $F(x)=\psi\left(-\psi^{-1}(x)\right)$, for some $\mathcal{C}^{k}$-diffeomorphism $\psi$, see for instance [11]. The main goal of this paper is to extend this result to dimension $n=2$ and prove the $\mathcal{C}^{k}$ version of Kerékjártó theorem. A first step in this direction can be found in [4], where we dealt with $\mathcal{C}^{1}$-involutions. Here we prove:

Theorem A. Let $F: \mathbb{R}^{2} \rightarrow \mathbb{R}^{2}$ be a $\mathcal{C}^{k}$-differentiable m-periodic map with $k \in\{1,2, \ldots, \infty\}$. Then $F$ is $\mathcal{C}^{k}$-linearizable.

This result cannot be extended to arbitrary dimension because in the papers $[5,8,10]$ it is shown that for $n \geq 7$ there are smooth periodic maps without fixed points. Obviously these maps cannot be linearized. It is worth to remark that, when dealing with local linearizability, there is a result valid in arbitrary dimension. Montgomery and Bochner proved that in any dimension, $m$-periodic maps having a fixed point and of class $\mathcal{C}^{k}, k \geq 1$, are always locally $\mathcal{C}^{k}$-linearizable in a neighborhood of this point, see [14] or Theorem 3.8 below. As we will see, an extension of this result will be one of the clues in our approach.

The proof of Theorem A is done in several steps. To describe them, let us introduce some notation. Given a m-periodic $\mathcal{C}^{k}$-map $F$ we can apply the classical Kerékjártó theorem and we already know that it is $\mathcal{C}^{0}$-conjugated to:

- The symmetry $S(x, y)=(x,-y)$ in the orientation reversing case,

- The rotation $R_{m}^{j}$, where $R_{m}$ is the rotation of angle $\alpha=2 \pi / m$ and $j$ is coprime with $m$, in the orientation preserving case.

We want to show that if $F$ is of class $\mathcal{C}^{k}$ then it is possible to find a $\mathcal{C}^{k}$-conjugation. Notice that in the orientation preserving case it suffices to consider the case of a $\mathcal{C}^{0}$-conjugacy with $R_{m}$, because the other cases can be obtained by iteration. In fact when $F$ is conjugated to $R_{m}^{j}$ and $p j+q m=1$, then $F^{p}$ is conjugated to $R_{m}$ and $F=\left(F^{p}\right)^{j}$.

For $i=1, \ldots, m$, denote the rays starting at the origin by

$$
K_{i}:=\left\{r\left(\cos \frac{2(i-1) \pi}{m}, \sin \frac{2(i-1) \pi}{m}\right): r \geq 0\right\} .
$$

Then we consider $A_{m}=\cup_{i=1}^{m} K_{i}$ and we call it the $m$-star. This $m$-star is a sort of skeleton of our map and the strategy to prove Theorem A will be developped in three steps, in each of them we conjugate $F$ with a map having a contact with the linear map $R$ near $A_{m}$, where $R=R_{m}$ when $m>2$, and $R \in\left\{R_{2}, S\right\}$, when $m=2$. The order of this contact is improved in each step. More concretely, we prove that the $m$-periodic $\mathcal{C}^{k}$-map $F$ is $\mathcal{C}^{k}$-conjugated with some new $m$-periodic maps $G_{1}, G_{2}$ and $G_{3}$ having $A_{m}$ as invariant set and satisfying: 
- First step: $\left.G_{1}\right|_{A_{m} \cup \mathcal{U}}=R$, where $\mathcal{U}$ is a neighborhood of the origin.

- Second step: $\left.G_{2}\right|_{A_{m} \cup \mathcal{U}}=R$ and $\left.d\left(G_{2}\right)\right|_{A_{m} \cup \mathcal{U}}=R$, where $\mathcal{U}$ is a neighborhood of the origin.

- Third step: $G_{3} \mid \mathcal{V}=R$, where $\mathcal{V}$ is a neighborhood of $A_{m}$.

Finally the $\mathcal{C}^{k}$-conjugation between $G_{3}$ and $R$ is easily constructed by gluing certain diffeomorphisms defined on the open sectors of $\mathbb{R}^{2} \backslash A_{m}$. As we will see, the resulting map is smooth precisely because $G_{3}$ coincides with $R$ in a neighborhood of $A_{m}$. In the previous discussion we have unified the cases of maps preserving or reversing orientation but many times in the course of the proof it will be convenient to study them separately.

The rest of the paper is divided in six sections and an appendix. Some applications of Theorem A are presented in Section 2. In Section 3 we collect some known results which will become useful tools later. In particular we state the so-called Smoothing Theorem, that allows to modify a piecewise smooth homeomorphism in order to produce a diffeomorphism. This result is proved in Hirsch's book [9] in the $\mathcal{C}^{\infty}$ context. We will also need some refinements and a $\mathcal{C}^{k}$ version with $k<\infty$. The proof is substantially different in these cases and we have included all the details in the Appendix. The tools introduced by Munkres in his book [16] will be crucial. The next three sections of the paper are devoted to prove each of the steps of the proof we described above. The last section of the paper contains the proofs of the results stated in Section 2. $\mathcal{C}^{k}$

Throughout the paper $k$ takes any value in $\{1,2, \ldots, \infty\}$ and all diffeomorphisms are of class

\section{Some consequences of Theorem A}

Recall that linear Floquet theory allows to transform non-autonomous $T$-periodic linear differential equations $x^{\prime}=A(t) x$ into autonomous linear differential equations $y^{\prime}=B y$, by using a $T$-periodic non-autonomous change of variables. As a first application of Theorem A we state the following result that can be interpreted as a Floquet type result for nonlinear equations, see Section 7.1 for a proof.

Corollary 2.1. Let $X: \mathbb{R} \times \mathbb{R}^{2} \longrightarrow \mathbb{R}^{2}$ be a time dependent $\mathcal{C}^{\infty}$-vector field such that $X(t+T, x)=$ $X(t, x)$ for all $(t, x) \in \mathbb{R} \times \mathbb{R}^{2}$. Assume that every solution of

$$
\frac{d x}{d t}=X(t, x)
$$

is periodic with a period rationally dependent with $T$. Then there exists a $\mathcal{C}^{\infty}, T$-periodic time dependent change of variables that transforms (1) into the linear differential system $\dot{y}=\frac{2 n \pi}{m T} A y$ where

$$
A=\left(\begin{array}{cc}
0 & 1 \\
-1 & 0
\end{array}\right)
$$

and $n, m \in \mathbb{N}$. In particular all orbits have a common period $m T$.

By a change of variables $x=\psi(t, y)$ we understand a $\mathcal{C}^{\infty}$-map $\psi: \mathbb{R} \times \mathbb{R}^{2} \rightarrow \mathbb{R}^{2}$ satisfying $\psi(t+T, y)=\psi(t, y)$ and such that $\psi(t, \cdot)$ is a $\mathcal{C}^{\infty}$-diffeomorphism of $\mathbb{R}^{2}$. 
The above result does not seem to have a direct extension valid for arbitrary dimensions. In Section 7.1 we will construct a $\mathcal{C}^{\infty}$ vector field, $X: \mathbb{R} \times \mathbb{R}^{7} \rightarrow \mathbb{R}^{7}$ which is $T$-periodic in $t$ and such that all the solutions of (1) are periodic with period $15 T$ but none of them has period $T$. In this case there is no change of variables $x=\psi(t, y)$ transforming (1) to a linear system $y^{\prime}=L y$ in $\mathbb{R}^{7}$, for otherwise the solution $x(t)=\psi(t, 0)$ should have period $T$. We do not know if the above Theorem admits an extension to some higher dimension $d$ with $3 \leq d \leq 6$

As a second application we study a class of stable fixed points for analytic area preserving maps that reverse orientation. Let $\mathcal{D}$ be an open and connected subset of the plane and let $h: \mathcal{D} \subset \mathbb{R}^{2} \rightarrow \mathbb{R}^{2}$ be a real analytic map satisfying

$$
\operatorname{det} h^{\prime}(x)=-1 \text { for each } x \in \mathcal{D} \text {. }
$$

This condition implies that our map is a local diffeomorphism preserving area and reversing orientation.

A fixed point $x_{*}=h\left(x_{*}\right)$ is called stable if given any neighborhood $\mathcal{U}$ there exists another neighborhood $\mathcal{V}$ such that all forward iterates $h^{n}(\mathcal{V}), n \geq 0$, are well defined and contained in $\mathcal{U}$. As an example consider the symmetry $S: \mathbb{R}^{2} \rightarrow \mathbb{R}^{2}, S\left(x_{1}, x_{2}\right)=\left(x_{1},-x_{2}\right)$ having the origin $x_{*}=0$ as a stable fixed point. It was proved in [18] that this is essentially the only possible example. This means that any map in the above conditions and having a stable fixed point must be conjugate to $S$. The proof in [18] was based on Kerékjártó theorem and so the conjugacy was realized via homeomorphisms. Now we can improve the conclusion in [18] using our main result.

Corollary 2.2. Assume that $h: \mathcal{D} \subset \mathbb{R}^{2} \rightarrow \mathbb{R}^{2}$ is a real analytic map satisfying the condition (2) and having a stable fixed point $x_{*}$. Then there exist an invariant neighborhood $\mathcal{W} \subset \mathcal{D}$ of $x_{*}$ and a $C^{\infty}$-diffeomorphism $\psi: \mathcal{W} \rightarrow \mathbb{R}^{2}$ such that $\psi \circ h=S \circ \psi$ in $\mathcal{W}$. Moreover, if $\mathcal{D}=\mathbb{R}^{2}$ then we can take $\mathcal{W}=\mathbb{R}^{2}$.

Again we refer to Section 7.1 for a proof. We do not know if the map $\psi$ can be chosen as an analytic real diffeomorphism. Also, it is convenient to notice that the result in [18] was stated in a slightly more restricted framework. There it was assumed that $\mathcal{D}=\mathbb{R}^{2}$ and $h$ was a real analytic diffeomorphism of the whole plane.

\section{Some preliminary results}

We start stating three preliminary results. The first one asserts that any local diffeomorphism can be extended to be a global diffeomorphism, see [15].

Theorem 3.1. Let $M$ be a differentiable manifold and let $g: \mathcal{V} \rightarrow g(\mathcal{V}) \subset M$ be a diffeomorphism defined on a neighborhood $\mathcal{V}$ of a point $p \in M$. Then there exists a diffeomorphism $f: M \rightarrow M$ such that $\left.f\right|_{\mathcal{W}}=\left.g\right|_{\mathcal{W}}$ for some neighborhood $\mathcal{W} \subset \mathcal{V}$ of $p$.

The second result is the one we talked about in the introduction. Part (a) is given in [9] for $\mathcal{C}^{\infty}$-manifolds. Here we state a slightly modified version of the theorem for $\mathcal{C}^{k}$-manifolds that is proved in the appendix. 
Theorem 3.2. (a) Let $W_{0}$ and $W_{1}$ be two manifolds without boundary of dimension $n$ and assume that they can be decomposed in the form $W_{i}=M_{i} \cup N_{i}, i=0,1$, where $M_{i}$ and $N_{i}$ are closed $n$ dimensional sub-manifolds satisfying

$$
M_{i} \cap N_{i}=\partial M_{i}=\partial N_{i}=V_{i} .
$$

In addition assume that $h: W_{0} \rightarrow W_{1}$ is a homeomorphism mapping $\mathcal{C}^{k}$-diffeormophically $M_{0}$ onto $M_{1}$ and $N_{0}$ onto $N_{1}$. Then there exists a $\mathcal{C}^{k}$-diffeomorphism $f: W_{0} \rightarrow W_{1}$ such that

$$
f\left(M_{0}\right)=M_{1}, f\left(N_{0}\right)=N_{1} \text { and } f=h \text { on } V_{0} .
$$

Moreover $f$ can be chosen in such a way that it coincides with $h$ outside a given neighborhood of $V_{0}$.

(b) In addition assume that $\mathcal{G}$ is an open subset of $W_{0}$ such that $h$ is a $C^{k}$-diffeomorphism from $\mathcal{G}$ onto $h(\mathcal{G})$. Let $\mathcal{E}$ be another open subset of $W_{0}$ having a clean crossing with $V_{0}$ and a compact closure $\operatorname{cl}(\mathcal{E})$ contained in $\mathcal{G}$. Then $f$ can be constructed with the additional property $f=h$ on $\mathcal{E}$.

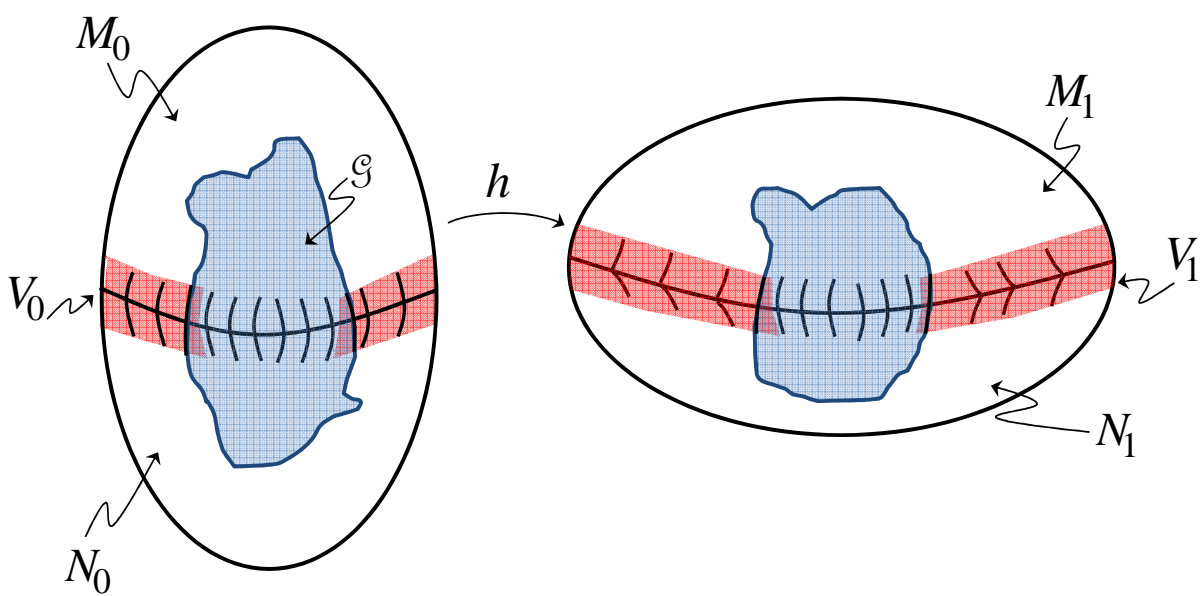

Figure 1: An illustration of the assumptions of Theorem 3.2 (b).

Notice that part (b) essentially says that when $h$ is smooth on some subset $\mathcal{G}$ of $V_{0}$, then it is not necessary to modify $h$ inside some prescribed compact region contained in $\mathcal{G}$. The above statement is incomplete because we have not defined the meaning of the term clean crossing. Given an open subset $\mathcal{E}$ of $W_{0}$, we say that $\mathcal{E}$ has a clean crossing with $V_{0}$ if there exist two sets $\mathcal{U}$ (open subset of $W_{0}$ with $V_{0} \subset \mathcal{U}$ ) and $E \subset V_{0}$ (open in the relative topology) and a $C^{k}$-diffeomorphism $\left.p: \mathcal{U} \rightarrow V_{0} \times\right]-1,1[$ satisfying

$$
p(x)=(x, 0), \text { if } x \in V_{0}, \quad \mathcal{U} \cap \mathcal{E}=p^{-1}(E \times]-1,1[) .
$$

To illustrate this definition we consider an example which will play a role later. Assume that $W_{0}=\mathbb{R}^{2} \backslash\{0\}$ and $V_{0}=\left(K_{1} \cup \cdots \cup K_{m}\right) \backslash\{0\}$ is the punctured $m$-star with $m$ even. The manifold $W_{0}$ 
is decomposed as the union of $M_{0}$ and $N_{0}$, where $M_{0}$ is the union of the angular sectors determined by the rays $K_{1}$ and $K_{2}, K_{3}$ and $K_{4}$ and so forth while the manifold $N_{0}$ has sectors determined by $K_{2}$ and $K_{3}, K_{4}$ and $K_{5} \ldots$ It is easy to prove that any annulus $\mathcal{E}=\left\{z \in \mathbb{R}^{2}: \delta<|z|<\Delta\right\}$ with $0<\delta<\Delta$ has a clean crossing with $V_{0}$.

The third result is a direct corollary of the natural generalization for non-compact $\mathcal{C}^{k}$-surfaces of the theorem of classification of $\mathcal{C}^{\infty}$-compact surfaces given in [9].

Theorem 3.3. Let $M$ be a simply connected and non-compact $\mathcal{C}^{k}$-surface such that $\partial M$ is connected and non-empty. Then $M$ is $\mathcal{C}^{k}$-diffeomorphic to $H=\left\{(x, y) \in \mathbb{R}^{2}: x \geq 1\right\}$.

The following semi-local version of the inverse function theorem will be used several times throughout the paper.

Theorem 3.4. Assume that $W_{0}$ and $W_{1}$ are $C^{k}$ manifolds of the same dimension, $A \subset W_{0}$ is a closed set, $\mathcal{U}$ is an open neighborhood of $A$ and $g: \mathcal{U} \longrightarrow W_{1}$ is a $\mathcal{C}^{k}$-map. Assume that $\left.g\right|_{A}$ is an homeomorphism between $A$ and $g(A)$ and $\operatorname{det}(d(g))_{x} \neq 0$ for each $x \in A$. Then there exists an open set $\mathcal{V} \subset W_{0}$ such that $A \subset \mathcal{V} \subset \mathcal{U}$ and $g: \mathcal{V} \longrightarrow g(\mathcal{V})$ is a $\mathcal{C}^{k}$-diffeomorphism.

This result can be obtained as a direct consequence of a beatiful topological result due to Munkres (Lemma 5.7 in [16]):

Theorem 3.5. Assume that $X$ and $Y$ are separable metric spaces and $X$ is locally compact. Let $A \subset X$ be a closed set and let $g: X \longrightarrow Y$ be a continuous map whose restriction becomes a homeomorphism between $A$ and $g(A)$. In addition, for each point $x$ of $A$ there is an open neighborhood which is mapped homeomorphically by $g$ onto an open subset of $Y$. Then there exists an open set $\mathcal{V}$ such that $A \subset \mathcal{V} \subset \mathcal{U}$ and $g: \mathcal{V} \longrightarrow g(\mathcal{V})$ is a homeomorphism.

To deal with the first step of our proof we also need to prove the following improvement of the Theorem 2.4 of [4].

Lemma 3.6. Let $C$ be a closed, connected and non-compact $\mathcal{C}^{k}$-submanifold of $\mathbb{R}^{2}$. Then there exists a diffeomorphism $\varphi: \mathbb{R}^{2} \rightarrow \mathbb{R}^{2}$ such that $\varphi(C)=\{0\} \times \mathbb{R}$. Moreover, assume that $C$ is such that there exists a global $\mathcal{C}^{k}$-parametrization $\gamma: \mathbb{R} \longrightarrow \mathbb{R}^{2}$ of $C$ such that $\gamma(y)=(0, y)$ for all $y \in(-a, a)$, where $a>0$. Then $\varphi$ can be chosen such that $\left.\varphi\right|_{\mathcal{V}}=\operatorname{Id}$ for some neighborhood $\mathcal{V}$ of the origin.

By a closed, connected and non-compact $C^{k}$-submanifold of $\mathbb{R}^{2}$ we mean a $C^{k}$ curve which is unbounded and closed as a subset of $\mathbb{R}^{2}$. With some work it can be proved that these curves can be characterized by the existence of a $C^{k}$ bijective parametrization $\gamma: \mathbb{R} \longrightarrow C$ with $\gamma^{\prime}(t) \neq 0$ for each $t \in \mathbb{R}$ and $\lim _{|t| \rightarrow \infty}\|\gamma(t)\|=\infty$.

Proof. The first statement is proved in Theorem 2.4 of [4] for the case $k=1$. The result in the general case follows by the same arguments and using Theorems 3.2 and 3.3 instead of their corresponding $\mathcal{C}^{1}$ versions.

We look now for the second statement. Consider the orthogonal unitary vector field given by $X(\gamma(t))=N\left(\frac{\gamma^{\prime}(t)}{\left\|\gamma^{\prime}(t)\right\|}\right)$ where $N(x, y)=(y,-x)$. Then $X$ is a $\mathcal{C}^{k-1}$ - vector field transversal to the curve $C$. Let $Y$ be a unitary $\mathcal{C}^{k}$-vector field transversal to the curve $C$ obtained by perturbing $X$. 
We can choose $Y$ in such a way $Y(0, y)=(1,0)$ for all $y \in(-b, b)$ and $0<b<a$. We are going to define a map $\pi: \mathbb{R}^{2} \longrightarrow \mathbb{R}^{2}, \pi(s, t)=(x, y)$, extending the parameterization $t \mapsto \gamma(t)$. More precisely

$$
\pi(s, t)=\gamma(t)+s Y(\gamma(t)) .
$$

This is a $C^{k}$ map and we will prove that it is also an embedding when it is restricted to an appropriate neighborhood of $s=0$. This will be our first opportunity to apply Theorem 3.4. Set $M=\mathbb{R}^{2}$ and $A=\{0\} \times \mathbb{R}$, then the restriction of $\pi$ to $A$ is a homeomorphism onto the curve $C$. Moreover the differential along $A$ can be expressed as a $2 \times 2$ matrix with columns

$$
d(\pi)(0, t)=(Y(\gamma(t)) \mid \dot{\gamma}(t))
$$

and the transversality of $Y$ and $C$ implies that the corresponding determinant does not vanish. Then we find an open set $\mathcal{W} \subset \mathbb{R}^{2}$ such that $\{0\} \times \mathbb{R} \subset \mathcal{W}$ and $\pi: \mathcal{W} \longrightarrow \pi(\mathcal{W})$ is a $C^{k}$ diffeomorphism. Now we choose a $C^{k}$-map $\sigma: \mathbb{R} \rightarrow(0,1)$ such that

$$
B=\left\{(s, t) \in \mathbb{R}^{2}:|s|<\sigma(t)\right\}
$$

is contained in $\mathcal{W}$. We will work with the restricted diffeomorphism $\pi: B \longrightarrow \pi(B)$. It satisfies

$$
\pi(0, t)=\gamma(t) \text { if } t \in \mathbb{R} \text { and } \pi(s, t)=(s, t) \text { if }|t|<b,|s|<\sigma(t) .
$$
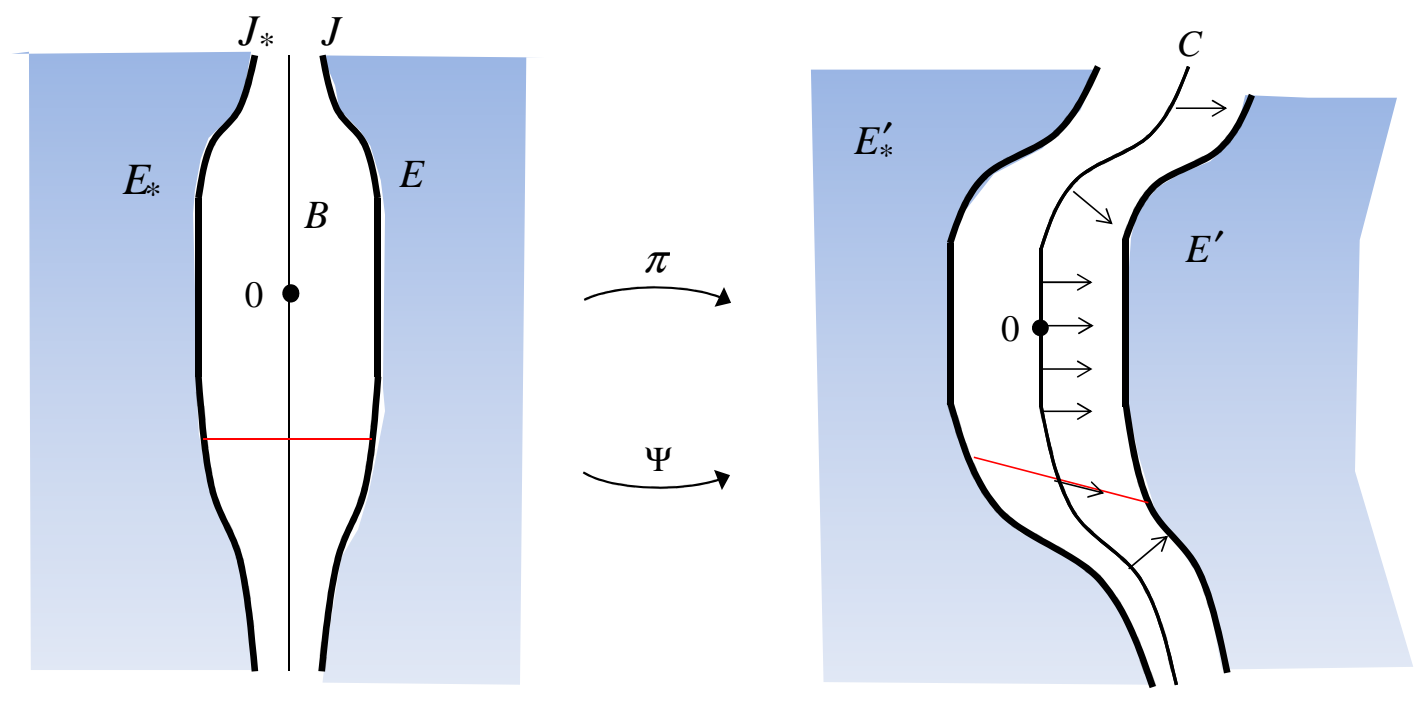

Figure 2: $\mathcal{C}^{k}$-diffeomorphisms, $\pi: B \rightarrow \pi(B), \psi: E \rightarrow E^{\prime}$.

Set $E=\left\{(s, t) \in \mathbb{R}^{2}: s \geq \frac{\sigma(t)}{2}\right\}$ and denote by $J=\left\{(s, t) \in \mathbb{R}^{2}: s=\frac{\sigma(t)}{2}\right\}$ which is a closed $\mathcal{C}^{k}$-submanifold of $\mathbb{R}^{2}$ contained in $B$. Since $\pi$ is a diffeomorphism it follows that $\pi(J)$ is also a $\mathcal{C}^{k}$ submanifold of $\mathbb{R}^{2}$, parameterized by $\Gamma(t)=\gamma(t)+\frac{\sigma(t)}{2} Y(\gamma(t))$. Since $\|\gamma(t)\| \rightarrow \infty$ as $|t| \rightarrow \infty$, we also have that $\|\Gamma(t)\| \rightarrow \infty$ and so $\pi(J)$ is a closed subset of $\mathbb{R}^{2}$. The set $\pi(J) \cup\{\infty\}$ is a Jordan 
curve lying inside $\mathbb{S}^{2}=\mathbb{R}^{2} \cup\{\infty\}$, then it divides the plane in two connected components. The curves $\{0\} \times \mathbb{R}$ and $J$ are disjoint and the same holds for the images under $\pi, C$ and $\pi(J)$. Let $D$ be the connected component that does not intersect $C$ and set $E^{\prime}=D \cup \pi(J)$. From Theorem 3.3 it follows that both $E^{\prime}$ and $E$ are both $\mathcal{C}^{k}$-diffeomorphic to $H=\left\{(x, y) \in \mathbb{R}^{2}: x \geq 1\right\}$. Therefore they are $\mathcal{C}^{k}$-diffeomorphic. Let $\psi: E \longrightarrow E^{\prime}$ be a $\mathcal{C}^{k}$-diffeomorphism between them. Then $\psi(J)=\pi(J)$. Now we want to modify $\psi$ in such a way that the new map coincides with $\pi$ on $J$.

To do this consider the map $\Delta: J \longrightarrow J$ defined as

$$
\Delta(z)=\psi^{-1}(\pi(z))
$$

which is a diffeomorphism. Therefore there exists a diffeomorphism $g: \mathbb{R} \longrightarrow \mathbb{R}$ such that $\Delta\left(\frac{\sigma(t)}{2}, t\right)=\left(\frac{\sigma(g(t))}{2}, g(t)\right)$. Thus the map $\Omega: E \longrightarrow E$ given by

$$
\Omega(s, t)=\left(s+\frac{\sigma(g(t))-\sigma(t)}{2}, g(t)\right)
$$

is a diffeomorphism and it satisfies that $\left.\Omega\right|_{J}=\Delta$. This map is obtained as $\Omega=\psi^{-1} \circ G \circ \psi$ where $G(s, t)=(s, g(t))$ and $\psi(s, t)=\left(s-\frac{\sigma(t)}{2}, t\right)$. The composition $h_{+}=\psi \circ \Omega: E \longrightarrow E^{\prime}$ is a diffeomorphism that satisfies $\left.(\psi \circ \Omega)\right|_{J}=\left.\pi\right|_{J}$.

In a symmetric way we construct a diffeomorphism $h_{-}: E_{*} \longrightarrow E_{*}^{\prime}$ where $E_{*}=\left\{(s, t) \in \mathbb{R}^{2}\right.$ : $\left.s \leq-\frac{\sigma(t)}{2}\right\}, E_{*}^{\prime}=D_{*} \cup \pi\left(J_{*}\right), J_{*}$ is the line of equation $s=-\frac{\sigma(t)}{2}$ and $D_{*}$ is the component of $\mathbb{R}^{2} \backslash \pi\left(J_{*}\right)$ which does not contain $C$. It satisfies $h_{-}=\pi$ on $J_{*}$.

Lastly set $h: \mathbb{R}^{2} \longrightarrow \mathbb{R}^{2}$ given by

$$
h= \begin{cases}h_{+}, & \text {on } E \\ \pi, & \text { on } B \\ h_{-}, & \text {on } E_{*} .\end{cases}
$$

It is a homeomorphism in the conditions of Theorem 3.2 with $W_{0}=W_{1}=\mathbb{R}^{2}, V_{0}=J \cup J_{*}$, $M_{0}=E \cup E_{*}, N_{0}=B$. Therefore we can find a $C^{k}$ diffeomorphism $\Psi: \mathbb{R}^{2} \longrightarrow \mathbb{R}^{2}$ which coincides with $h$ outside a prescribed neighborhood of $V_{0}$. This neighborhood can be chosen so that its closure is disjoint with the line $\{0\} \times \mathbb{R}$. Then $\Psi=\pi$ on a neighborhood of the vertical axis. In particular $\Psi$ equals the identity on a neighborhood of the segment $\{0\} \times[-b, b]$. Thus $\Psi^{-1}$ is the desired diffeomorphism.

Remark 3.7 Notice that if $C_{1}$ and $C_{2}$ are closed and non compact submanifolds and $\gamma_{1}, \gamma_{2}$ are parametrizations of $C_{1}$ and $C_{2}$ satisfying $\gamma_{1}(s)=\gamma_{2}(s)$ for all $s \in J, J$ open subset of $\mathbb{R}$, then the difeomorphisms $\varphi_{1}$ and $\varphi_{2}$ obtained in the above Lemma can be chosen so that they coincide in a neighborhood of $\gamma_{1}(J)$.

Finally we recall the classical Montgomery-Bochner theorem ([14]).

Theorem 3.8. (Montgomery-Bochner theorem). Let $\mathcal{U} \subset \mathbb{R}^{n}$ be an open set and let $F: \mathcal{U} \rightarrow \mathcal{U}$ be a class $\mathcal{C}^{k}, k \geq 1$, m-periodic map, having a fixed point $p \in \mathcal{U}$ and let $L=d(F)_{p}$ be the differential of $F$ at $p$. Then the map $\psi=\frac{1}{m} \sum_{i=0}^{m-1} L^{-i} \circ F^{i}$ conjugates $F$ and $L$ in a neighborhood of $p$. 
Nowadays the above map $\psi$ is called the Montgomery-Bochner transformation. It can be seen it is not necessarily a global conjugation, see for instance [3]. It may be convenient to be precise on the meaning of the local conjugacy in the above result. Indeed the identity $\psi \circ F=L \circ \psi$ holds everywhere but $\psi$ is a diffeomorphism from $\mathcal{V}$ onto $\psi(\mathcal{V})$, where $\mathcal{V}$ is a neighborhood of the origin which can be chosen invariant under $F$.

\section{First step: Conjugacy with a map having the $m$-star fixed}

The goal of this section is to prove the following proposition, that in the orientation preserving case allows to linearize $F$ on the $m$-star and near the origin.

Proposition 4.1. Let $F: \mathbb{R}^{2} \rightarrow \mathbb{R}^{2}$ be a m-periodic $\mathcal{C}^{k}$-map which is $\mathcal{C}^{0}$-conjugate to $R_{m}$. Then $F$ is $\mathcal{C}^{k}$-conjugate to a map $G: \mathbb{R}^{2} \rightarrow \mathbb{R}^{2}$ that has $A_{m}$ as invariant set and satisfies $G=R_{m}$ on $A_{m} \cup \mathcal{U}$, where $\mathcal{U}$ is some neighborhood of the origin.

Proof. It is divided in four steps.

Step 1. There exists a map $\widetilde{F}: \mathbb{R}^{2} \rightarrow \mathbb{R}^{2}$ which is $\mathcal{C}^{k}$-conjugate to $F$ and coincides with $R_{m}$ on some neighborhood of the origin.

By assumption $F$ and $R_{m}$ are topologically conjugate and so $F$ has a unique fixed point. Moreover we know by Theorem 3.8 that $F$ is locally conjugate (in $\mathcal{C}^{k}$ ) to its linear part $L$. Hence $R_{m}$ and $L$ are also locally conjugate (in $\mathcal{C}^{k}$ ) and, being linear, they are conjugate in the linear sense. After a linear change of variables we can assume $L=R_{m}$. Let $\varphi: \mathcal{W} \longrightarrow \mathbb{R}^{2}$ be a $\mathcal{C}^{k}$-map that conjugates $F$ to $R_{m}$ in a neighborhood $\mathcal{W}$ of $p$. From Theorem 3.1 the embedding $\varphi$ can be extended to be a global diffeomorphism $\pi: \mathbb{R}^{2} \rightarrow \mathbb{R}^{2}$ such that $\left.\pi\right|_{\mathcal{V}}=\left.\varphi\right|_{\mathcal{V}}$ for some neighborhood $\mathcal{V} \subset \mathcal{W}$ of $p$. Since $F$ is topologically conjugate to $R_{m}$ we can select $\mathcal{V}$ so that $F(\mathcal{V})=\mathcal{V}$. Consider now $\widetilde{F}=\pi \circ F \circ \pi^{-1}$. The map $\widetilde{F}$ has 0 as a fixed point and in the neighborhood of the origin $\pi(\mathcal{V}), \widetilde{F}$ is exactly $R_{m}$.

Next we introduce some topological notions that will play a role in the second step. An arc $\gamma$ from 0 to $\infty$ is the image of a continuous and one-to-one map $f:[0, \infty) \rightarrow \mathbb{R}^{2}$ satisfying $f(0)=0$ and $\lim _{t \rightarrow \infty}\|f(t)\|=\infty$. Given two arcs $\gamma_{1}$ and $\gamma_{2}$ with $\gamma_{1} \cap \gamma_{2}=\{0\}$, the set $\gamma_{1} \cup \gamma_{2} \cup\{\infty\}$ is a Jordan curve lying in $\mathbb{S}^{2}=\mathbb{R}^{2} \cup\{\infty\}$ and Schonflies theorem implies that $\mathbb{R}^{2} \backslash\left(\gamma_{1} \cup \gamma_{2}\right)$ has two connected components, both homeomorphic to the open disk.

A topological $m$-star $\mathcal{A}=\cup_{i=1}^{m} \mathcal{K}_{i}$ will be composed by $m$ arcs $\mathcal{K}_{i}$ from 0 to $\infty$ satisfying $\mathcal{K}_{i} \cap \mathcal{K}_{j}=\{0\}$ if $i \neq j, \mathbb{R}^{2} \backslash \mathcal{A}$ has $m$ components and the component $\mathcal{B}_{i}$ is characterized by two properties: its boundary is the union of $\mathcal{K}_{i}$ and $\mathcal{K}_{i+1}$ and the remaining arcs are disjoint with $\mathcal{B}_{i}$. In practice we will simply say that $\mathcal{A}$ is a star while $A_{m}$ will be distinguished as the standard star. Step 2. There exists a star $\bar{L}=\cup_{i=1}^{m} \bar{L}_{i}$ satisfying

$\bar{L} \cap B=A_{m} \cap B$ for some closed ball $B$ centered at the origin

$\bar{L} \backslash\{0\}$ is a $C^{k}$ manifold $\bar{L}_{i}=\widetilde{F}^{(i-1)}\left(\bar{L}_{1}\right), i=2, \ldots, m$.

Let $\psi: \mathbb{R}^{2} \rightarrow \mathbb{R}^{2}$ be the homeomorphism such that $\psi^{-1} \circ \widetilde{F} \circ \psi=R_{m}$. Consider the $m$-star $A_{m}$ and let $L:=\psi\left(A_{m}\right)=\cup_{i=1}^{m} \psi\left(K_{i}\right)$. Then $L$ is a star invariant under $\widetilde{F}$. Furthermore, if we denote by $\widetilde{K}_{i}:=\psi\left(K_{i}\right)$ we get that $\widetilde{K}_{i}=\widetilde{F}^{i}\left(\widetilde{K}_{0}\right)$. This is not the star we are looking for since it is probably non smooth and we do not have any information around the origin. For this reason we 
are going to rearrange the rays $\widetilde{K}_{i}$. To this end, let $b>0$ be such that the closed ball $B_{b}(0) \subset \pi(\mathcal{V})$. Consider the the last point of $\widetilde{K}_{1}$ meeting $B_{b}(0)$. Here the orientation of the arc $\widetilde{K}_{1}$ going from 0 to $\infty$ has been selected. In principle this point can be anywhere on the circumference of radius $b$ but, after rotating the coordinate system, we can assume that it is precisely the point $(b, 0)$.

Let us do some surgery: we remove from $\widetilde{K}_{1}$ the arc between the origin and $(b, 0)$ and substitute it by the segment joining these two points. We denote the obtained arc by $L_{1}^{\prime}$ and by $L_{i}^{\prime}:=$ $\widetilde{F}^{(i-1)}\left(L_{1}^{\prime}\right)$. Now the set $L^{\prime}:=\cup_{i=1}^{m} L_{i}^{\prime}$ is again a star invariant under $\widetilde{F}$ and $L^{\prime} \cap B_{b}(0)=A_{m} \cap B_{b}(0)$. This is not yet the searched star because it is not necessarily smooth. Denote by $B_{1}^{\prime}$ the connected component of $\mathbb{R}^{2} \backslash L^{\prime}$ adjacent to $L_{1}^{\prime}$ and $L_{2}^{\prime}$. This set can be seen as one of the connected components of $\mathbb{S}^{2} \backslash \Gamma$ where $\Gamma$ is the Jordan curve composed by the two arcs and the point of infinity. In consequence $\infty$ is accessible from $B_{1}^{\prime}$ and we can find an $\operatorname{arc} \bar{L}_{1}=f([0, \infty))$ joining 0 and $\infty$ satisfying the following properties:

(i) $f(t)=(t, 0)$ if $t \leq b / 2$,

(ii) $f(t) \in B_{1}^{\prime}$ and $\|f(t)\|>\frac{b}{2}$ for all $t>b / 2$,

(iii) $f$ is $\mathcal{C}^{k}$ and $f^{\prime}(t) \neq 0$ for all $t \geq 0$.

Defining $\bar{L}:=\cup_{i=1}^{m} \widetilde{F}^{i}\left(\bar{L}_{1}\right)$ we have the searched star. Step 3. There exists a map $\bar{G}: \mathbb{R}^{2} \longrightarrow \mathbb{R}^{2}$ satisfying

- $\bar{G}$ is $C^{k}$ conjugated to $\widetilde{F}$

- $\bar{G}=R_{m}$ in a neighborhood of the origin

- $\bar{G}\left(K_{i}\right)=K_{i+1}, i=1, \ldots, m$ where $K_{i}$ are the rays of the standard star $A_{m}$

To do this let $g: \mathbb{R} \longrightarrow \mathbb{R}$ be a degree one $\mathcal{C}^{k}$-map, (i.e. $\left.g(\theta+2 \pi)=g(\theta)+2 \pi\right)$ ) such that $g(0)=-\pi / 2, g\left(\frac{2 \pi}{m}\right)=\pi / 2$ and $g^{\prime}(x)>0$ for all $x \in \mathbb{R}$. Then the map $H: \mathbb{R}^{2} \longrightarrow \mathbb{R}^{2}$ which in polar coordinates is given by $H(r, \theta)=(r, g(\theta))$ is a homeomorphism of $\mathbb{R}^{2}$ and the restriction to $\mathbb{R}^{2} \backslash\{(0,0)\}$ is a diffeomorphism.

Consider now $T=H\left(\bar{L}_{1} \cup \bar{L}_{2} \cup\{(0,0)\}\right.$ which is a $\mathcal{C}^{k}$-closed submanifold. Now we are in the conditions of Lemma 3.6 and we get a diffeomorphism $h: \mathbb{R}^{2} \longrightarrow \mathbb{R}^{2}$ verifying $h(T)=\{0\} \times \mathbb{R}$ and $\left.h\right|_{\mathcal{V}}=$ Id in some neighborhood $\mathcal{V}$ of the origin, see Figure 3. Thus the map $\psi_{1}:=H^{-1} \circ h \circ H$ is a diffeomorphism on $\mathbb{R}^{2} \backslash\{(0,0)\}$ and a homeomorphism on $\mathbb{R}^{2}$. Furthermore, since it is the identity in a neighborhood of the origin, we conclude that $\psi_{1}$ is a diffeomorphism of $\mathbb{R}^{2}$.

In addition it has the following properties:

$$
\psi_{1}\left(\bar{L}_{1}\right)=K_{1} \quad, \quad \psi_{1}\left(\bar{L}_{2}\right)=K_{2} \quad \text { and } \quad \psi_{1}\left(\bar{B}_{1}\right)=B_{1},
$$

where we denote by $B_{i}$ the connected component of $\mathbb{R}^{2} \backslash A_{m}$ adjacent to $K_{i}$ and $K_{i+1}$.

Doing a similar process we obtain a diffeomorphism $\widetilde{\psi}_{2}: \mathbb{R}^{2} \longrightarrow \mathbb{R}^{2}$ such that it is the identity in a neighborhood of the origin, and

$$
\widetilde{\psi}_{2}\left(\bar{L}_{2}\right)=K_{2} \quad, \quad \widetilde{\psi}_{2}\left(\bar{L}_{3}\right)=K_{3} \quad \text { and } \quad \widetilde{\psi}_{2}\left(\bar{B}_{2}\right)=B_{2} .
$$




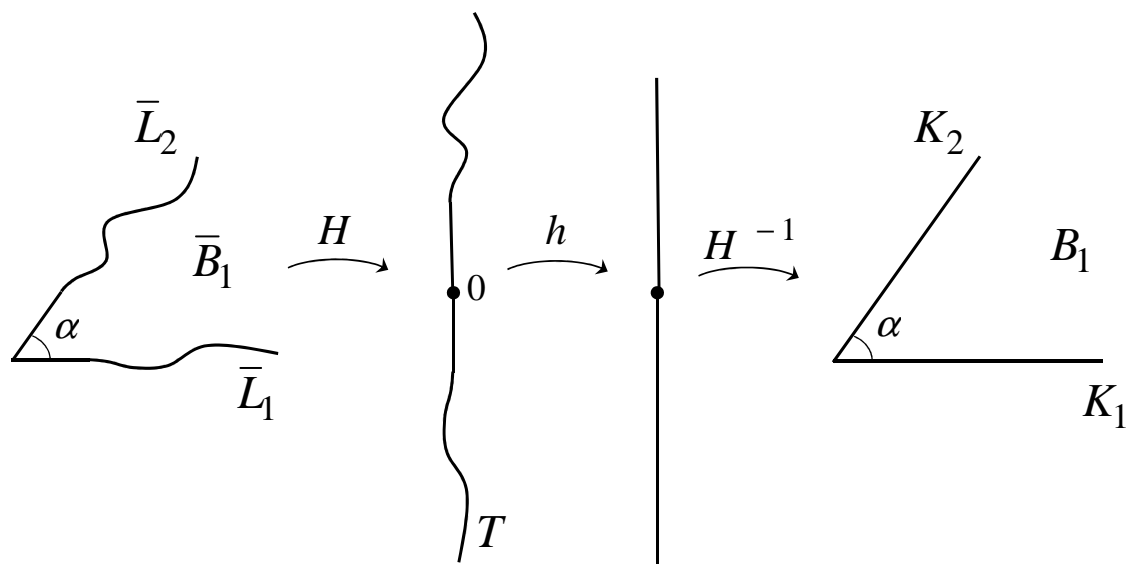

Figure 3: Construction of $\psi_{1}:=H^{-1} \circ h \circ H$ to regularize one sector of the topological star $\bar{L}$.

Now we want to modify $\widetilde{\psi}_{2}$ in such a way that it coincides with $\psi_{1}$ at $\bar{L}_{2}$. Since $\psi_{1}\left(\bar{L}_{2}\right)=\widetilde{\psi}_{2}\left(\bar{L}_{2}\right)=$ $K_{2}$ the map $p_{2}:=\psi_{1} \circ \widetilde{\psi}_{2}^{-1}: K_{2} \longrightarrow K_{2}$ is given by an one dimensional diffeomorphism which is the identity near of the origin. Thus we can consider the map $\widetilde{p}_{2}: \mathbb{R}^{2} \longrightarrow \mathbb{R}^{2}$ that in polar coordinates is given by $\widetilde{p}_{2}(r, \theta)=\left(p_{2}(r), \theta\right)$. Since $p_{2}$ is a diffeomorphism that begins being the Identity, it follows that $\widetilde{p}_{2}$ is a diffeomorphism of $\mathbb{R}^{2}$ that is the Identity in a neighborhood of the origin. Now consider $\psi_{2}:=\widetilde{p}_{2} \circ \widetilde{\psi}_{2}$. Then $\psi_{2}$ is a diffeomorphism such that it is the identity in a neighborhood of the origin, maps $\bar{L}_{i}$ onto $K_{i}$ for $i=2,3, \psi_{2}\left(\bar{B}_{2}\right)=B_{2}$ and coincides with $\psi_{1}$ on $\bar{L}_{2}$. Proceeding in this way we obtain diffeomorphisms $\psi_{1}, \psi_{2}, \ldots, \psi_{m-1}$.

To construct a diffeomorphism $\psi_{m}$ such that $\psi_{m}\left(\bar{B}_{m}\right)=B_{m},\left.\psi_{m}\right|_{\bar{L}_{m}}=\left.\psi_{m-1}\right|_{\bar{L}_{m}}$ and $\left.\psi_{m}\right|_{\bar{L}_{1}}=$ $\left.\psi_{1}\right|_{\bar{L}_{1}}$ we proceed in a slightly different way. First we construct $\widetilde{\psi}_{m}: \mathbb{R}^{2} \longrightarrow \mathbb{R}^{2}$ in an analogous way as $\widetilde{\psi}_{2}$ was constructed. After that the map $p_{m}:=\psi_{m-1} \circ \widetilde{\psi}_{m}^{-1}: K_{m} \longrightarrow K_{m}$ is a diffeomorphism that begins with the Identity. In a similar way the map $p_{1}:=\psi_{1} \circ \widetilde{\psi}_{m}^{-1}: K_{1} \longrightarrow K_{1}$ has the same property. Thus we define $\widetilde{p}_{m}: \mathbb{R}^{2} \longrightarrow \mathbb{R}^{2}$ that in polar coordinates is given by

$$
\widetilde{p}_{m}(r, \theta)=\left(a(\theta) p_{m}(r)+(1-a(\theta)) p_{1}(r), \theta\right) \text { with } a(0)=0, a\left(\frac{2(m-1) \pi}{m}\right)=1
$$

where $a: \mathbb{R} \rightarrow[0,1]$ is a $2 \pi$-periodic function in $\mathcal{C}^{k}$. Since both $p_{1}$ and $p_{m}$ begin with the Identity we get that for $r$ small enough $\widetilde{p}_{m}(r, \theta)=(r, \theta)$. Thus $\widetilde{p}_{m}$ is also a diffeomorphisms of $\mathbb{R}^{2}$ that is the identity in a neighborhood of the origin. Lastly we define $\psi_{m}=\widetilde{p}_{m} \circ \widetilde{\psi}_{m}$ that has the desired properties.

Now let $\widetilde{\varphi}: \mathbb{R}^{2} \longrightarrow \mathbb{R}^{2}$ defined by $\widetilde{\varphi}(z)=\psi_{i}(z)$ when $z \in \bar{B}_{i} \cup \bar{L}_{i} \cup \bar{L}_{i+1}$. Thus $\widetilde{\varphi}$ is an homeomorphism that restricted to each piece $\bar{B}_{i} \cup \bar{L}_{i} \cup \bar{L}_{i+1} \backslash\{(0,0)\}$ is a diffeomorphism and is the identity near the origin. Thus we can apply parts (a) and (b) of Theorem 3.2 to obtain a diffeomorphism $\phi: \mathbb{R}^{2} \longrightarrow \mathbb{R}^{2}$ that is the identity near the origin and $\phi(\bar{L})=A_{m}$. Therefore the map $\bar{G}=\phi \circ \widetilde{F} \circ \phi^{-1}$ is obviously $\mathcal{C}^{k}$-conjugated to $\widetilde{F}$, has $A_{m}=\phi(\bar{L})$ as invariant set and near the origin coincides with $R_{m}$. It is perhaps worth to give more details on the way Theorem 3.2 is applied to construct $\phi^{-1}$. When $m$ is even we take $W_{0}=W_{1}=\mathbb{R}^{2} \backslash\{0\}, V_{0}=A_{m} \backslash\{0\}$, 
$V_{1}=\bar{L} \backslash\{0\}, M_{0}=\cup_{i \text { odd }} B_{i}, N_{0}=\cup_{i \text { even }} B_{i}$. The set $\mathcal{G}$ is any open neighborhood of the origin where $\widetilde{\varphi}$ equals the identity and $\mathcal{E}$ is an annulus $0<r<|z|<R$ whose closure is contained in $\mathcal{G}$. We observe that the map given by the Theorem can be modified in the disk $|z| \leq r$ in order to get a diffeomorphism which coincides with identity in a neigborhood of the origin. When $m$ is odd we must proceed in two steps. First we apply the Theorem with $W_{0}=B_{1} \cup B_{2} \cup\left(L_{2} \backslash\{0\}\right), V_{0}=L_{1}$ and then we apply it again with $W_{0}=\mathbb{R}^{2} \backslash\{0\}$ and $V_{0}=L_{1} \cup L_{3} \cup \cdots \cup L_{m}$.

Step 4. Conclusion.

To finish the proof of the proposition we only need to conjugate in $\mathcal{C}^{k}$ the map $\bar{G}$ to a map $G$ preserving the properties of $\bar{G}$ and additionally satisfying that $G=R_{m}$ on $K_{i}$ for all $i=$ $0,1, \ldots, m-1$.

We start with a modification of $\bar{G}$ satisfying this condition on $K_{1}$. Since $\bar{G}\left(K_{1}\right)=K_{2}$ the map $\left.\bar{G}\right|_{K_{1}}$ is given in polar coordinates by $\bar{G}(r, 0)=\left(f(r), \frac{2 \pi}{m}\right)$ for some diffeomorphism $f$ of $[0, \infty)$ that is the identity for $r$ small enough. Thus as in the previous reduction we now consider the map that in polar coordinates writes as $\Delta(r, \theta)=(b(\theta) f(r)+(1-b(\theta)) r, \theta)$ where $b: \mathbb{R} \rightarrow[0,1]$ is a $2 \pi$-periodic function in $\mathcal{C}^{k}$ satisfying

$$
b(0)=0, \quad b\left(\frac{2 \pi}{m}\right)=1 \quad \text { and } \quad b\left(\frac{2 \pi i}{m}\right)=0, i=2,3, \ldots, m-1 .
$$

Then the map $G=\Delta^{-1} \circ \bar{G} \circ \Delta$ satisfies $G(r, 0)=\left(r, \frac{2 \pi}{m}\right)$. Hence $G$ satisfies all the required properties on $K_{1}$ and does not change the map on $K_{i}, i \neq 1$. In a similar way we can continue the procedure arranging $G$ on the remaining rays. Note that in the penultimate step we already know that $G=R_{m}$ on $A_{m} \backslash K_{m}$. Then, we do not need to impose $G=R_{m}$ on $K_{m}$ because this is a direct consequence of $G^{m}=$ Id. This identity holds because $G$ and $R_{m}$ are conjugate.

\section{Second step: smooth matching with $R_{m}$ on the $m$-star}

The main result of this section is the following proposition. Notice that it only deals with the orientation preserving case and allows to obtain a map, $\mathcal{C}^{k}$-conjugated to $F$ and that has a $\mathcal{C}^{1}$ contact with $R_{m}$ on the $m$-star $A_{m}$. Remember that in Proposition 4.1 the contact was only of class $\mathcal{C}^{0}$.

Proposition 5.1. Let $F: \mathbb{R}^{2} \longrightarrow \mathbb{R}^{2}$ be a m-periodic $\mathcal{C}^{k}$-map, $\mathcal{C}^{0}$-conjugate to $R_{m}$. Then $F$ is $\mathcal{C}^{k}$-conjugate to a m-periodic map $G$ that has $A_{m}$ as invariant set and satisfies $G=R_{m}$ on $A_{m} \cup \mathcal{U}$, for some neighborhood $\mathcal{U}$ of the origin, and moreover $d(G)_{(x, y)}=R_{m}$ for each $(x, y) \in A_{m}$.

To prove it we will use the following result, already given for the case $k=1$ in $[4$, Lem. 2.3]. The proof for the general case follows exactly the same arguments as in that paper, by using Theorems 3.2 and 3.3 instead of their corresponding $\mathcal{C}^{1}$ versions.

Lemma 5.2. Let $D \subset \mathbb{R}^{2}$ be an open and simply connected set such that $\{0\} \times \mathbb{R} \subset D$. Then there exist an open set $\mathcal{V}$ such that $\{0\} \times \mathbb{R} \subset \mathcal{V} \subset D$ and a diffeomorphism $\psi: D \rightarrow \mathbb{R}^{2}$ such that $\left.\psi\right|_{\mathcal{V}}=\mathrm{Id}$.

Corollary 5.3. Let $C$ be a closed and non-compact $\mathcal{C}^{k}$-submanifold of $\mathbb{R}^{2}, \mathcal{U}$ an open neighborhood of $C$ and $g: \mathcal{U} \longrightarrow \mathbb{R}^{2}$ such that $g$ is a diffeomorphism onto its image and $g(C)$ is closed in $\mathbb{R}^{2}$. Then there exists a diffeomorphism $\psi: \mathbb{R}^{2} \longrightarrow \mathbb{R}^{2}$ such that $\left.\psi\right|_{\mathcal{V}}=\left.g\right|_{\mathcal{V}}$ for some open neighborhood $\mathcal{V}$ of $C$ contained in $\mathcal{U}$. 
Proof. Without loss of generality we can assume that $\mathcal{U}$ is simply connected. Let $\varphi_{1}, \varphi_{2}: \mathbb{R}^{2} \longrightarrow \mathbb{R}^{2}$ be the diffeomorphisms given by Lemma 3.6 such that $\varphi_{1}(C)=\varphi_{2}(g(C))=\{0\} \times \mathbb{R}$. Applying Lemma 5.2 to $\varphi_{1}(\mathcal{U})$ and $\varphi_{2}\left(g(\mathcal{U})\right.$ ) we obtain diffeomorphisms $\psi_{1}: \varphi_{1}(\mathcal{U}) \longrightarrow \mathbb{R}^{2}$ and $\psi_{2}: \varphi_{2}(g(\mathcal{U})) \longrightarrow \mathbb{R}^{2}$ that are the identity in suitable neighborhoods of $\{0\} \times \mathbb{R}$. Therefore we obtain the desired diffeomorphism by considering $\varphi_{2}^{-1} \circ \psi_{2} \circ \varphi_{2} \circ g \circ \varphi_{1}^{-1} \circ \psi_{1}^{-1} \circ \varphi_{1}$.

Next result also plays a key role in our approach. From now on, $G L_{2}(\mathbb{R})$ will denote the group of linear automorphisms of $\mathbb{R}^{2}$.

Theorem 5.4. Let $C \subset \mathbb{R}^{2}$ be a closed and non compact $\mathcal{C}^{k}$-submanifold. Let $\gamma: \mathbb{R} \longrightarrow C$ be a $\mathcal{C}^{k}$-parametrization ${ }^{1}$ of $C$ and $\psi: \mathbb{R} \longrightarrow G L_{2}(\mathbb{R})$ a $\mathcal{C}^{k-1}$ map satisfying that

$$
\psi(y)\left(\gamma^{\prime}(y)\right)=\gamma^{\prime}(y) \text { for all } y \in \mathbb{R} .
$$

Then there exists a diffeomorphism $\Psi: \mathbb{R}^{2} \longrightarrow \mathbb{R}^{2}$ such that

$$
\left.\Psi\right|_{C}=\operatorname{Id} \text { and } d(\Psi)_{\gamma(y)}=\psi(y) \text { for all } y \in \mathbb{R} .
$$

If in addition $\psi(y)=\mathrm{Id}$ for all $y \in J, J$ open subset of $\mathbb{R}$, then $\Psi$ can be chosen in such a way that $\left.\Psi\right|_{\mathcal{V}}=\operatorname{Id}$ in some neighborhood $\mathcal{V}$ of $\gamma(J)$.

Proof. First we prove the result in the case that $C=\{0\} \times \mathbb{R}$ and $\gamma(y)=(0, y)$. In this situation

$$
\psi(y)=\left(\begin{array}{ll}
A(y) & 0 \\
B(y) & 1
\end{array}\right)
$$

for some $A, B: \mathbb{R} \longrightarrow \mathbb{R}, \mathcal{C}^{k-1}$-maps with $A(y) \neq 0$ for all $y \in \mathbb{R}$. Now consider the map $H: \mathbb{R}^{2} \longrightarrow \mathbb{R}^{2}$ given by

$$
H(x, y)=\left(\int_{y}^{y+x} A(s) d s, y+\int_{y}^{y+x} B(s) d s\right) .
$$

Clearly $H$ is of class $\mathcal{C}^{k}, H(0, y)=(0, y)$ and $d(H)_{(0, y)}=\left(\begin{array}{cc}A(y) & 0 \\ B(y) & 1\end{array}\right)$. Since $\left(\operatorname{det}\left(d(H)_{(0, y)}\right)=\right.$ $A(y) \neq 0$ we can apply Corollary 3.4 and we obtain that $\left.H\right|_{\mathcal{U}}$ is a diffeomorphism for a certain neighborhood $\mathcal{U}$ of $\{0\} \times \mathbb{R}$ that we can choose simply connected. Now the result follows by applying Corollary 5.3 to $\{0\} \times \mathbb{R}$ and $\left.H\right|_{\mathcal{U}}$. Observe that if $\psi(y)=\operatorname{Id}$ for all $y \in J$ then $A(y)=1$ and $B(y)=0$ for all $y \in J$ and therefore $H(x, y)=(x, y)$ in a suitable neighborhood of $\{0\} \times J$. This ends the proof of the Theorem in this case.

Now we turn to the general case. From Lemma 3.6 there exists a diffeomorphism $\pi: \mathbb{R}^{2} \longrightarrow \mathbb{R}^{2}$ such that $\pi(\{0\} \times \mathbb{R})=C$. After some modifications following previous ideas we can even assume that $\pi(0, y)=\gamma(y)$. Then consider the $\mathcal{C}^{k-1}$ map $\Delta: \mathbb{R} \longrightarrow G L_{2}(\mathbb{R})$ given by

$$
\Delta(y)=d\left(\pi^{-1}\right)_{\pi(0, y)} \circ \psi(y) \circ d(\pi)_{(0, y)} .
$$

\footnotetext{
${ }^{1}$ in particular $\gamma^{\prime} \neq 0$ everywhere
} 
By differentiating the identity $\pi(0, y)=\gamma(y)$ we get that $t_{y}=d(\pi)_{(0, y)}((0,1))=\gamma^{\prime}(y)$ is a tangent vector to $C$ at the point $\pi(0, y)=\gamma(y)$. Moreover, by hypothesis, $\psi(y)\left(\gamma^{\prime}(y)\right)=\gamma^{\prime}(y)$. Therefore we get:

$$
\Delta(y)(0,1)=d\left(\pi^{-1}\right)_{\pi(0, y)}\left(\psi(y)\left(d(\pi)_{(0, y)}\right)\right)(0,1)=d\left(\pi^{-1}\right)_{\pi(0, y)}\left(t_{y}\right)=(0,1) .
$$

Thus we obtain a diffeomorphism $\widetilde{\Psi}: \mathbb{R}^{2} \longrightarrow \mathbb{R}^{2}$ such that $\left.\widetilde{\Psi}\right|_{\{0\} \times \mathbb{R}}=\operatorname{Id}$ and $d(\widetilde{\Psi})_{(0, y)}=\Delta(y)$. Now the result follows by considering $\Psi=\pi \circ \widetilde{\Psi} \circ \pi^{-1}$.

Remark 5.5 Notice that if $C_{1}, \gamma_{1}, \psi_{1}$ and $C_{2}, \gamma_{2}, \psi_{2}$ satisfy the hypotheses of the theorem and, for some open subset $J$ of $\mathbb{R}, \gamma_{1}(t)=\gamma_{2}(t)$ and $\psi_{1}(t)=\psi_{2}(t)$ for all $t \in J$ then the diffeomorphisms $\Psi_{1}$ and $\Psi_{2}$ obtained in the above theorem coincide in a neighborhood of $\gamma_{1}(J)$. The proof of this assertion combines Remark 3.7 with the previous proof. In particular the following observation plays a role: $\psi_{1}=\psi_{2}$ on $J$ implies that $A_{1}=A_{2}, B_{1}=B_{2}$ on the same set $J$.

Assume now that in addition to the previous conditions on $\gamma_{1}$ and $\gamma_{2}, \psi_{1}$ and $\psi_{2}$, we know that $\psi_{1}(t)=$ Id if $t \in J_{*}$, where $J_{*}$ is an open subset of $\mathbb{R}$. Then we can construct $\Psi_{1}$ and $\Psi_{2}$ satisfying simultaneously $\Psi_{1}=\Psi_{2}$ in a neighborhood of $\gamma_{1}(J)$ and $\Psi_{1}=\operatorname{Id}$ in a neighborhood of $\gamma_{1}\left(J_{*}\right)$.

Now we are ready to go ahead with the second step in the proof of theorem A.

Proof of Proposition 5.1. From Proposition 4.1 we can assume that $F$ satisfies all required properties except that $d(F)_{(x, y)}=R_{m}$ if $(x, y) \in A_{m}$. Denote by $G L_{2}(\mathbb{R})^{*}$ be the subgroup of $G L_{2}(\mathbb{R})$ of the automorphisms having $(1,0)$ as an eigenvector of eigenvalue 1 . We recall that $\alpha=2 \pi / m$ and define the vectors $v_{i}=(\cos i \alpha, \sin i \alpha)$ for each $i \in\{0, \ldots, m-1\}$. From $F=R_{m}$ on $A_{m}$ we get that $R_{m}^{-1} F\left(x v_{i}\right)=x v_{i}$ for each $x>0$ and differentiating this identity,

$$
d\left(R_{m}^{-1} \circ F\right)_{x v_{i}}\left(v_{i}\right)=v_{i} .
$$

Since $v_{i}=R_{m}^{i}(1,0)$ we obtain

$$
\left(R_{m}^{-(i+1)} \circ d(F)_{x v_{i}} \circ R_{m}^{i}\right)(1,0)=(1,0) .
$$

For each $i=0,1, \ldots, m-1$ we define $\Lambda_{i}(x)$ as the matrix

$$
\Lambda_{i}(x):=R_{m}^{-(i+1)} \circ d(F)_{x v_{i}} \circ R_{m}^{i} .
$$

Then $\Lambda_{i}(x) \in G L_{2}(\mathbb{R})^{*}$ and

$$
d(F)_{x v_{i}}=R_{m}^{(i+1)} \circ \Lambda_{i}(x) \circ R_{m}^{-i}
$$

We claim that the automorphisms $\Lambda_{i}$ satisfy

(i) $\Lambda_{i}(x)=$ Id for $x$ small enough, and

(ii) $\Lambda_{m-1}(x) \circ \ldots \circ \Lambda_{1}(x) \circ \Lambda_{0}(x)=$ Id for all $x>0$.

Statement (i) follows from the fact that $F=R_{m}$ in a neighborhood of the origin. To prove (ii) we see that

$$
\mathrm{Id}=d\left(F^{m}\right)_{x v_{0}}=d(F)_{x v_{m-1}} \circ \ldots \circ d(F)_{x v_{1}} \circ d(F)_{x v_{0}}=\Lambda_{m-1}(x) \circ \ldots \circ \Lambda_{1}(x) \circ \Lambda_{0}(x) .
$$


This ends the proof of the claim.

Now we look for a diffeomorphism $\varphi$ satisfying that $\left.\varphi\right|_{A_{m}}=\operatorname{Id},\left.d\left(\varphi^{-1} \circ F \circ \varphi\right)\right|_{A_{m}}=R_{m}$ and $\left.\varphi\right|_{\mathcal{U}}=\mathrm{Id}$ for a certain neighborhood $\mathcal{U}$ of the origin. Direct computations show that if such diffeomorphism exists it must satisfy

$$
d(\varphi)_{x v_{i+1}}=d(F)_{x v_{i}} \circ d(\varphi)_{x v_{i}} \circ R_{m}^{-1} .
$$

Thus choosing $d(\varphi)_{x v_{0}}=\mathrm{Id}$, we obtain inductively

$$
d(\varphi)_{x v_{i}}=R_{m}^{i} \circ \Lambda_{i-1}(x) \circ \ldots \circ \Lambda_{1}(x) \circ \Lambda_{0}(x) \circ R_{m}^{-i} .
$$

To obtain such a diffeomorphism we proceed as follows. If we denote by $B_{k}$ the connected component of $\mathbb{R}^{2} \backslash A_{m}$ adjacent to the rays $K_{k}$ and $K_{k+1}$ we want to construct a diffeomorphism $\varphi_{k}$ defined in a neighborhood of $B_{k}$ that sends $B_{k}$ onto itself, is the identity in a neighborhood of the origin and satisfies equation (3) for $i=k$. Moreover we want that $\varphi_{k}$ and $\varphi_{k+1}$ coincide in a neighborhood of $K_{k+1}$. We will see that we will obtain the desired difeomorphism $\varphi$ by gluing the corresponding diffeomorphisms $\left.\varphi_{k}\right|_{B_{k}}$.

Let us start the construction of $\varphi$. Let $r$ be small enough such that $\left.F\right|_{B_{r}(0)}=R_{m}, \bar{K}_{i}:=$ $K_{i} \cap\left(\mathbb{R}^{2} \backslash B_{r / 2}(0)\right)$ and let $\gamma_{i}$ be a compact $\mathcal{C}^{k}$-arc joining the points $\frac{r}{2} v_{i-1}$ and $\frac{r}{2} v_{i}$ and such that the open arc is contained in $B_{i} \cap B_{r / 2}(0)$ and the curve $D_{i}:=\bar{K}_{i} \cup \gamma_{i} \cup \bar{K}_{i+1}$ is $\mathcal{C}^{k}$.

Now we have that $D_{1}$ is a closed and non-compact $\mathcal{C}^{k}$-submanifold. We parametrize it by the map $l_{1}: \mathbb{R} \longrightarrow \mathbb{R}^{2}$ given by

$$
l_{1}(x)= \begin{cases}-x v_{0} & \text { if } x \leq-r / 2 \\ \delta_{1}(x), & \text { if } x \in[-r / 2, r / 2] \\ x v_{1} & \text { if } x \geq r / 2\end{cases}
$$

where $\delta_{1}:[-r / 2, r / 2] \longrightarrow \mathbb{R}^{2}$ is a $\mathcal{C}^{k}$-parametrization of $\gamma_{1}$ chosen such that $l_{1}$ is also a $\mathcal{C}^{k}-$ parametrization.

Consider the $\mathcal{C}^{k-1}$ map $\Phi_{1}: \mathbb{R} \longrightarrow G L_{2}(\mathbb{R})$ given by

$$
\Phi_{1}(x)= \begin{cases}\mathrm{Id}, & \text { if } x \leq r / 2 \\ R_{m} \circ \Lambda_{0}(x) \circ R_{m}^{-1}, & \text { if } x \geq r / 2 .\end{cases}
$$

Now we can apply Theorem 5.4 with $C=D_{1}, \gamma=l_{1}$ and $\psi=\Phi_{1}$. Thus we obtain a difeomorphism

$$
\varphi_{1}: \mathbb{R}^{2} \longrightarrow \mathbb{R}^{2} \text { such that }\left.\varphi_{1}\right|_{D_{1}}=\mathrm{Id} \text { and } d\left(\varphi_{1}\right)_{l_{1}(x)}=\Phi_{1}(x) .
$$

Moreover since $\Phi_{1}(x)=$ Id for all $x<r$ we get that $\varphi_{1}$ is the identity in a neighborhood of $\bar{K}_{1} \cup \gamma_{1}$, see Figure 4.

In a similar way can parametrize $D_{2}$ by $l_{2}: \mathbb{R} \longrightarrow \mathbb{R}^{2}$ given by

$$
l_{2}(x)= \begin{cases}-x v_{2} & \text { if } x \leq-r / 2 \\ \delta_{2}(x), & \text { if } x \in[-r / 2, r / 2] \\ x v_{1} & \text { if } x \geq r / 2\end{cases}
$$




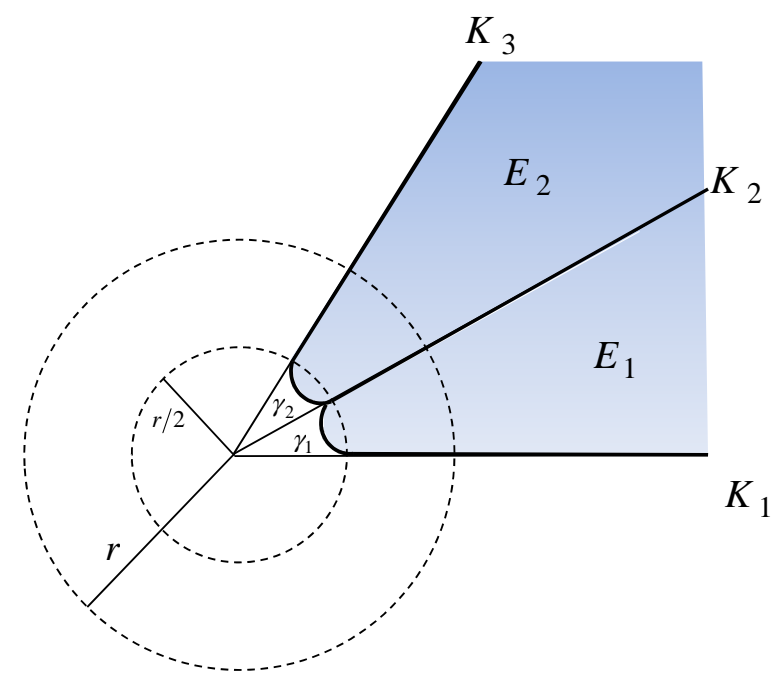

Figure 4: The $\mathcal{C}^{k}$-diffeomorphisms $\varphi_{1}$ and $\varphi_{2}$ are defined in such a way that coincide on an open neighborhood of the common boundary between $D_{1}$ and $D_{2}$ and are the identity in a neighborhood of the ball of radius $r / 2$.

and consider the $\mathcal{C}^{k-1}$ map $\Phi_{2}: \mathbb{R} \longrightarrow G L_{2}(\mathbb{R})$ given by

$$
\Phi_{2}(x)= \begin{cases}R_{m}^{2} \circ \Lambda_{1}(x) \circ \Lambda_{0}(x) \circ R_{m}^{-2}, & \text { if } x \leq-r / 2 ; \\ \mathrm{Id}, & \text { if } x \in[-r / 2, r / 2] ; \\ R_{m} \circ \Lambda_{0}(x) \circ R_{m}^{-1}, & \text { if } x \geq r / 2 .\end{cases}
$$

Applying again Theorem 5.4 with $C=D_{2}, \gamma=l_{2}$ and $\psi=\Phi_{2}$ we obtain a diffeomorphism

$$
\varphi_{2}: \mathbb{R}^{2} \longrightarrow \mathbb{R}^{2} \text { such that }\left.\varphi_{2}\right|_{D_{2}}=\mathrm{Id} \text { and } d\left(\varphi_{2}\right)_{l_{2}(x)}=\Phi_{2}(x) .
$$

Moreover, as on the first sector, since $\Phi_{2}(x)=\mathrm{Id}$ for all $\left.x \in\right]-r, r\left[\right.$ we get that $\varphi_{2}$ is the identity in a neighborhood of $\gamma_{2}$. Lastly since the parametrization of $\bar{K}_{2}$ given by $l_{1}$ and $l_{2}$ coincide, and $\Phi_{1}(x)=\Phi_{2}(x)$ when $x \in \bar{K}_{2}$ from Remark 5.5 we obtain that $\varphi_{1}$ and $\varphi_{2}$ also coincide in some neighborhood of $\bar{K}_{2}$, see again Figure 4 .

Iterating this procedure we obtain diffeomorphisms of $\mathbb{R}^{2}, \varphi_{1}, \varphi_{2}, \ldots, \varphi_{m}$ satisfying the following properties:
(a) $\left.\varphi_{i}\right|_{D_{i}}=\mathrm{Id}$,
(b) $\varphi_{i}$ is the identity in a neighborhood of $\gamma_{i}$,
(c) $\left.d\left(\varphi_{i}\right)\right|_{x v_{i-1}}=R_{m}^{i-1} \circ \Lambda_{i-2}(x) \circ \ldots \circ \Lambda_{1}(x) \circ \Lambda_{0}(x) \circ R_{m}^{-(i-1)}$,
(d) $\left.d\left(\varphi_{i}\right)\right|_{x v_{i}}=R_{m}^{i} \circ \Lambda_{i-1}(x) \circ \ldots \circ \Lambda_{1}(x) \circ \Lambda_{0}(x) \circ R_{m}^{-i}$,
(e) For $i=2 \ldots, m$ we have that $\varphi_{i-1}$ and $\varphi_{i}$ coincide in a neighborhood of $\bar{K}_{i}$. 
Moreover since $\Phi_{m}(x)=$ Id when $l_{m}(x) \in \bar{K}_{1}$ we also obtain that $\varphi_{m}=$ Id in a neighborhood of $\bar{K}_{1}$. Thus $\varphi_{m}$ and $\varphi_{1}$ also coincide in a neighborhood of $\bar{K}_{1}$.

Now denote by $E_{i}$ the closure of the connected component of $\mathbb{R}^{2} \backslash D_{i}$ contained in $B_{i}$ and consider $\varphi: \mathbb{R}^{2} \longrightarrow \mathbb{R}^{2}$ defined by

$$
\varphi(x)= \begin{cases}\varphi_{i}(x), & \text { if } x \in E_{i} \\ x, & \text { otherwise }\end{cases}
$$

Clearly $\varphi$ is a diffeomorphism and by construction satisfies that it is the identity in a neighborhood of the origin and that $\left.d\left(\varphi^{-1} \circ F \circ \varphi\right)\right|_{A_{m}}=R_{m}$, as we wanted to prove.

\section{Third step and proof of Theorem A}

We start proving next two propositions. The first one gives the third step of our approach in the orientation preserving case. The second one is the equivalent statement for the orientation reversing situation. We remark that the orientation reversing case has not been treated yet, therefore the proof of Proposition 6.1 deals simultaneously with the three steps of our approach.

Proposition 6.1. Let $F: \mathbb{R}^{2} \longrightarrow \mathbb{R}^{2}$ be a $\mathcal{C}^{k}$, m-periodic map, $\mathcal{C}^{0}$-conjugated to $R_{m}$. Then $F$ is $\mathcal{C}^{k}$-conjugated to a m-periodic map $G$ that coincides with $A_{m}$ in some neighborhood of $A_{m}$.

Proposition 6.2. Let $F: \mathbb{R}^{2} \rightarrow \mathbb{R}^{2}$ be a planar involution $\mathcal{C}^{0}$-conjugated to $S$. Then $F$ is $C^{k}$ conjugated to an involution $G$ that coincides with $S$ in some neighborhood of $A_{2}$.

Proof of Proposition 6.1. From Proposition 5.1 we can assume that $F$ has $A_{m}$ as invariant set $\left.F\right|_{A_{m}}=\left.R_{m}\right|_{A_{m}}, d(F)_{(x, y)}=R_{m}$ if $(x, y) \in A_{m}$ and $F=R_{m}$ in some neighborhood $\mathcal{U}$ of the origin.

Now consider the Montgomery-Bochner transformation $\psi=\frac{1}{m} \sum_{i=0}^{m-1} R_{m}^{-i} \circ F^{i}$. Clearly we have that $R_{m} \circ \psi=\psi \circ F$. On the other hand since $F=R_{m}$ on $A_{m}$ we have that $\left.\psi\right|_{A_{m}}=$ Id. Moreover since $d(F)_{(x, y)}=R_{m}$ if $(x, y) \in A_{m}$ we also have that $d(\psi)_{(x, y)}=$ Id. Note also that $\left.\psi\right|_{\mathcal{U}}=$ Id. Therefore from Corollary 3.4 we deduce that there exists $\mathcal{V}$, an open neighborhood of $A_{m}$, such that $\left.\psi\right|_{\mathcal{V}}$ is a diffeomorphism onto its image.

Now set $r>0$ such that $B_{r}(0) \subset \mathcal{U} \cap \mathcal{V}$. Now we repeat the construction given in the proof of the Proposition 5.1. That is, we consider $\mathcal{C}^{k}$-arcs $\gamma_{i}$ contained in $C_{i} \cap B_{r / 2}(0)$ joining the points $\frac{r}{2} v_{i}$ and $\frac{r}{2} v_{i+1}$ such that the curve $D_{i}:=\bar{K}_{i} \cup \gamma_{i} \cup \bar{K}_{i+1}$ is of class $\mathcal{C}^{k}$. By construction $\mathcal{V}$ is still and open neighborhood of $D_{i}$, for each $i=1, \ldots, m$, and hence by Corollary 5.3 we obtain diffeomorphisms $f_{i}: \mathbb{R}^{2} \longrightarrow \mathbb{R}^{2}$ and $\mathcal{V}_{i}$ neighborhoods of $D_{i}$ such that $f_{i}\left|\mathcal{V}_{i}=\psi\right| \mathcal{V}_{i}$.

Using the notation introduced in the proof of Proposition 5.1 we define a diffeomorphism $f: \mathbb{R}^{2} \longrightarrow \mathbb{R}^{2}$ by

$$
f(x)= \begin{cases}f_{i}(x), & \text { if } x \in E_{i} \\ x, & \text { otherwise }\end{cases}
$$

Since $\left.f_{i}\right|_{\mathcal{V}_{i}}=\psi$ and $f_{i+1} \mid \mathcal{V}_{i+1}=\psi$ it follows that $f_{i}$ and $f_{i+1}$ coincide in a neighborhood of $K_{i+1} \cap\left(\mathbb{R}^{2} \backslash B_{r / 2}(0)\right)$. On the other hand since $\gamma_{i} \subset \mathcal{U} \cap \mathcal{V}$ it follows that $f_{i}$ is the identity in a suitable neighborhood of $\gamma_{i}$. In particular $E_{i}$ is invariant under $f$. These facts show that $f$ is a well defined diffeomorphism. Note also that by construction $\left.f\right|_{\mathcal{W}}=\left.\psi\right|_{\mathcal{W}}$ for a suitable neighborhood $\mathcal{W}$ of $A_{m}$. 
Lastly set $G=f \circ F \circ f^{-1}$. Since $R_{m} \circ \psi=\psi \circ F$ and $\psi$ is a diffeomorphism in a neighborhood of $A_{m}$ it follows that in a suitable neighborhoofd of $A_{m}$ the following equality holds:

$$
R_{m}=\psi \circ F \circ \psi^{-1} \text {. }
$$

Since $f$ coincides with $\psi$ in a neighborhood of $A_{m}$ we obtain that $G=R_{m}$ in some neighborhood of $A_{m}$, as we wanted to prove.

Proof of Proposition 6.2. Let $g: \mathbb{R}^{2} \rightarrow \mathbb{R}^{2}$ be the homeomorphism given by Kerékjártó theorem, such that $F \circ g=g \circ S$. Then, since $g$ is a homeomorphism, we know that $M:=g\left(A_{2}\right)$ is a non-compact, closed and connected topological submanifold of $\mathbb{R}^{2}$ which is fixed by $F$. Recall that $A_{2}=\mathbb{R} \times\{0\}$. We claim that $M$ is a differentiable submanifold of $\mathbb{R}^{2}$. To do this we show that $M$ is locally the graph of a $\mathcal{C}^{k}$-function.

Let $(a, b) \in M$. Then $(a, b)$ is a fixed point of $F$ and $d(F)_{(a, b)}$ is conjugated to $S$. Then $d(F)_{(a, b)}-\mathrm{Id} \neq 0$. If we write $F=\left(F_{1}, F_{2}\right)$ this implies that at least one of the functions $F_{1}(x, y)-x$ and $F_{2}(x, y)-y$ has non-zero gradient at $(a, b)$. Assume for instance that $\frac{\partial\left(F_{1}(x, y)-x\right)}{\partial x}(a, b) \neq 0$. By the Implicit Function theorem there exist neighborhoods $\mathcal{V}$ of $(a, b)$ and $\mathcal{W}$ of $b$ and a $\mathcal{C}^{k}$-map $\psi: \mathcal{W} \rightarrow \mathbb{R}$ such that $M \cap \mathcal{W}=\{(\psi(t), t): t \in \mathcal{W}\}$. This proves the claim.

¿From Lemma 3.6 there exists a diffeomorphism $\phi: \mathbb{R}^{2} \longrightarrow \mathbb{R}^{2}$ such that $\phi(M)=\mathbb{R} \times\{0\}$. Thus the map $\widetilde{F}:=\phi \circ F \circ \phi^{-1}$ is $\mathcal{C}^{k}$-conjugated to $F$ and has $\mathbb{R} \times\{0\}$ as a manifold of fixed points. Thus $\widetilde{F}(x, 0)=(x, 0)$ and

$$
d(\widetilde{F})_{(x, 0)}=\left(\begin{array}{cc}
1 & B(x) \\
0 & A(x)
\end{array}\right)
$$

for some $A, B: \mathbb{R} \rightarrow \mathbb{R}$ of class $\mathcal{C}^{k-1}$. Moreover since $d(\widetilde{F})_{(x, 0)}$ must be conjugated to $S$ it follows that $A(x)=-1$ for all $x \in \mathbb{R}$.

¿From Theorem 5.4 there exists a diffeomorphism $\psi: \mathbb{R}^{2} \longrightarrow \mathbb{R}^{2}$ such that $\psi(x, 0)=(x, 0)$ for all $x \in \mathbb{R}$ and

$$
d(\psi)_{(x, 0)}=\left(\begin{array}{cc}
1 & -B(x) / 2 \\
0 & 1
\end{array}\right) .
$$

If we denote by $\bar{F}=\psi^{-1} \circ \widetilde{F} \circ \psi$ direct computations show that $\bar{F}(x, 0)=(x, 0)$ and $d(\bar{F})_{(x, 0)}=S$ for all $x \in \mathbb{R}$.

Lastly let $N:=\frac{1}{2}(\operatorname{Id}+S \circ \bar{F})$ be the associated Montgomery-Bochner transformation. Direct computations show that $N(x, 0)=(x, 0)$ and $d(N)_{(x, 0)}=\mathrm{Id}$ for all $x \in \mathbb{R}$. Therefore from Corollary 3.4 it follows that $N$ is a diffeomorphism in a neighborhood of $A_{2}$. Moreover from Corollary 5.3 there exists a diffeomorphism $\Psi: \mathbb{R}^{2} \longrightarrow \mathbb{R}^{2}$ that coincides with $N$ in a suitable neighborhood of $A_{2}$. Thus we obtain the result by considering $G=\Psi \circ \bar{F} \circ \Psi^{-1}$.

Proof of Theorem A. We begin with the orientation preserving case. As we said in the introduction we can reduce to the case that $F$ is $\mathcal{C}^{0}$-conjugated to $R_{m}$ with $m \geq 2$. From Proposition 6.1 we can assume that $F=R_{m}$ in some neighborhood $\mathcal{V}$ of $A_{m}$.

As before, for $i=1, \ldots, m$ we denote by $B_{i}$ the closure of the connected component of $\mathbb{R}^{2} \backslash A_{m}$ adjacent to $K_{i}$ and $K_{i+1}$. Since $F$ is a homeomorphism mapping $K_{i} \cup K_{i+1}$ onto $K_{i+1} \cup K_{i+2}$ we deduce that $F\left(B_{i}\right)=B_{i+1}$. Consider now the map $H: \mathbb{R}^{2} \longrightarrow \mathbb{R}^{2}$ defined by $H=R_{m}^{i-1} \circ F^{-(i-1)}$ in $B_{i}$. Note that the sets $B_{i}$ are invariant under $H$ and $H$ restricted to a suitable neighborhood of $A_{m}$ 
is the identity. Thus $H$ is a $\mathcal{C}^{k}$-diffeomorphism. Lastly by construction we have $R_{m} \circ H=H \circ F$. This ends the proof of the theorem in this case.

Now we turn to the orientation reversing case. From Proposition 6.2 we can assume that $\left.F\right|_{\mathcal{V}}=\left.S\right|_{\mathcal{V}}$ in some neighborhood of $A_{2}=\mathbb{R} \times\{0\}$. Now define

$$
H(x, y)= \begin{cases}(x, y) & \text { if } y \geq 0 \\ F(S(x, y)) & \text { if } y \leq 0\end{cases}
$$

Since $H$ is the identity in a neighborhood of $A_{2}$ it follows that $H$ is diffeomorphism. Also direct computations show that $F \circ H=H \circ S$. This ends the proof of the theorem.

\section{$7 \quad$ Proof of corollaries}

This section is devoted to prove the two corollaries of Theorem A stated in Section 2.

\subsection{Proof of Corollary 2.1}

Let $\varphi(t, x)$ be the solution of (1) passing through $x$ at time $t=0$, that is

$$
\frac{\partial \varphi}{\partial t}(t, x)=X(t, \varphi(t, x)), \quad \varphi(0, x)=x .
$$

Set $F: \mathbb{R}^{2} \longrightarrow \mathbb{R}^{2}$ the Poincaré map, defined by $F(x)=\varphi(T, x)$. By hypothesis for each $x \in \mathbb{R}^{2}$ there exists $m_{x} \in \mathbb{N}$ such that $F^{m_{x}}(x)=x$. That is $F$ is pointwise periodic. It is well-known (see $[7,13])$ that in this situation there exists $m \in \mathbb{N}$ such that $F^{m}=\mathrm{Id}$. That is all solutions are $m T$ periodic. From Theorem A there exists a $\mathcal{C}^{k}$-diffeomorphism $\Phi$ of $\mathbb{R}^{2}$ such that $F=\Phi \circ R_{\alpha} \circ \Phi^{-1}$, where $\alpha=2 n \pi / m$. Thus the change $y=\Phi(x)$ transforms our original system into a new system satisfying that the corresponding Poincaré map is exactly $R_{\alpha}$. Thus we can assume that our system satisfies that $F=R_{\alpha}$ and

$$
\varphi(t+T, x)=\varphi\left(t, R_{\alpha} x\right) .
$$

Now, define $\Psi(t, x)=\varphi\left(t, R_{\left(-\frac{\alpha t}{T}\right)} x\right)$, where $R_{\theta}$ denotes the rotation of angle $\theta$. Notice that

$$
\Psi(t+T, x)=\varphi\left(t+T, R_{\left(-\frac{\alpha(t+T)}{T}\right)} x\right)=\varphi\left(t, R_{\alpha}\left(R_{\left(-\frac{\alpha(t+T)}{T}\right)} x\right)\right)=\varphi\left(t, R_{\left(-\frac{\alpha t}{T}\right)} x\right)=\Psi(t, x),
$$

so $\Psi$ is $T$-periodic. Now consider the change of variables $x=\Psi(t, y)$. We will have

$$
X(t, x)=\dot{x}=\frac{\partial \varphi}{\partial t}\left(t, R_{\left(-\frac{\alpha t}{T}\right)} x\right)+\frac{\partial \varphi}{\partial x}\left(t, R_{\left(-\frac{\alpha t}{T}\right)} y\right)\left(\dot{R}_{\left(-\frac{\alpha t}{T}\right)} y+R_{\left(-\frac{\alpha t}{T}\right)} \dot{y}\right) .
$$

From $X=\frac{\partial \varphi}{\partial t}$ and $\operatorname{det}\left(\frac{\partial \varphi}{\partial x}\right) \neq 0$ we conclude that

$$
\dot{R}_{\left(-\frac{\alpha t}{T}\right)} y+R_{\left(-\frac{\alpha t}{T}\right)} \dot{y}=0
$$

and obtain

as we wanted to prove.

$$
\dot{y}=-R_{\left(-\frac{\alpha t}{T}\right)}^{-1} \dot{R}_{\left(-\frac{\alpha t}{T}\right)} y=\frac{\alpha}{T} A y,
$$




\subsection{Construction of the example in $\mathbb{R}^{7}$}

Following [8] one can find $\mathcal{C}^{\infty}$ periodic maps $F: \mathbb{R}^{7} \rightarrow \mathbb{R}^{7}$ without fixed points as soon as the period is not a power of a prime number. Hence the first possible periods are $6,15, \ldots$ We choose the period 15 to guarantee that $\operatorname{det} F^{\prime}>0$ so that $F$ is an orientation-preserving diffeomorphism. In that case $F$ is also diffeotopic to the identity (see Theorem 8.3.1 in [9]) and so it can be realized as the Poincaré map of some periodic and smooth vector field $X: \mathbb{R} \times \mathbb{R}^{7} \rightarrow \mathbb{R}^{7}$, see Theorem 8.2.1 in $[12]$.

\subsection{Proof of Corollary 2.2}

First we select a small neighborhood $\mathcal{W} \subset \mathcal{D}$ of $x_{*}$ with the following properties:

- $\mathcal{W}$ is open and simply connected

- $h(\mathcal{W})=\mathcal{W}$

- The restriction of $h$ to $\mathcal{W}$ is one-to-one.

This is possible thanks to the inverse function theorem and a well-known result on the existence of invariant neighborhoods (see [18] and the references therein). The same argument of the proof of [18] allow us to find a sequence $\left\{x_{n}\right\}$ of fixed points accumulating at $x_{*}$; that is,

$$
x_{n} \rightarrow x_{*}, \quad x_{n} \neq x_{*} \text { and } h\left(x_{n}\right)=x_{n} .
$$

These points are also fixed under $h^{2}=h \circ h$ and so $x_{*}$ is a non-isolated fixed point of $h^{2}$. Corollary 1 in [17] can be applied ${ }^{2}$ to the map $h^{2}: \mathcal{W} \rightarrow \mathbb{R}^{2}$. It implies that $h^{2}=$ Id in $\mathcal{W}$.

Next we transport our map via a $C^{\infty}$-diffeomorphism between $\mathcal{W}$ and $\mathbb{R}^{2}$. Note that there are many diffeomorphisms of this type. A way to construct examples is to use Riemann's theorem to map $\mathcal{W}$ conformally onto the open unit disk $\mathbb{D}$ and then to compose this map with a radial $C^{\infty}$-diffeomorphism between $\mathbb{D}$ and $\mathbb{R}^{2}$. Define $H=\varphi \circ h \circ \varphi^{-1}$. Then $H$ is a $C^{\infty}$-map satisfying $H^{2}=\mathrm{Id}$. Our main result applies and the conclusion is obtained after pulling back from $\mathbb{R}^{2}$ to $\mathcal{W}$. In the case $\mathcal{D}=\mathbb{R}^{2}$ the identity $h^{2}=\operatorname{Id}$ in $\mathcal{W}$ leads to $h^{2}=$ Id in $\mathbb{R}^{2}$ by analytic continuation. In this case the change of variables $\varphi$ is not employed.

\section{Appendix: Proof of Theorem 3.2}

The so-called Smoothing Theorem is an useful tool to transform piecewise smooth homeomorphisms into diffeomorphisms. It is stated and proved by Hirsch for $C^{\infty}$-manifolds in [9, p. 182]. The same result is also valid for manifolds of finite class and we will state it in this more general framework. As in the previous sections, manifolds, maps and diffeomorphisms will be understood in the class $C^{k}$, where $k=1,2, \ldots$ is fixed. The case $k=\infty$ is also included. All manifolds under consideration will be in particular topological spaces with a countable basis. In this appendix we prove Theorem 3.2. In [4] we stated part (a) of this result for the case $k=1$ and we referred to the book [9] for a proof. As previously mentioned, Hirsch's book deals with the case $k=\infty$, in the paper [4] we left to the

\footnotetext{
${ }^{2}$ the set $\mathcal{U}$ in the statement of this result must be connected
} 
reader the details of the passage from $k=\infty$ to $k=1$. This was probably misleading because the proof in [9] cannot be easily modified. Indeed there are at least two steps in that proof where there is a loss of derivatives. This is the case in the construction of isotopies via differential equations ([9, Chp. 8]) and also in the proposed isotopy between a map and its derivative at a point ([9, p. 112]). The loss of derivatives is irrelevant when working in $C^{\infty}$ but it would decrease the class of the diffeomorphism when one is working in a finite class. Fortunately the book by Munkres [16] contains all the tools needed for the proof of Theorem 3.2. The isotopies in Munkres' book are constructed carefully in order to preserve the level of differentiability. Next we present a proof based on [16] and [9], hoping that this time our patient readers will easily follow all the details. The terminology is taken from [9]. We start with a preliminary observation which will be employed several times and it is somehow linked with the definition of clean crossing. In a neighborhood of $V_{0}$ the manifolds $W_{0}, M_{0}$ and $N_{0}$ can be viewed as cylinders based on $V_{0}$. More precisely, it is possible to find an open set $\mathcal{U}$ in $W_{0}$ with $V_{0} \subset \mathcal{U}$ and a diffeomorphism $\left.p: \mathcal{U} \rightarrow V_{0} \times\right]-1,1[$ satisfying

$$
p(x)=(x, 0) \text { if } x \in V_{0}, \quad p\left(\mathcal{U} \cap M_{0}\right)=V_{0} \times\left[0,1\left[, \quad p\left(\mathcal{U} \cap N_{0}\right)=V_{0} \times\right]-1,0\right] .
$$

This is a direct consequence of the existence of tubular neighborhoods in the $\mathcal{C}^{\infty}$ case (see [16], page 53). However in the $\mathcal{C}^{k}$ case with $k<\infty$ the map $p$ constructed from the tubular neighborhood will be of class $\mathcal{C}^{k-1}$. Thus an alternative procedure has to be employed if we want to avoid the loss of one derivative. We explain it briefly. Given a $\mathcal{C}^{k}$-manifold $M$ with boundary $\partial M$, there exists a $\mathcal{C}^{k}$-diffeomorphism $p: \mathcal{U} \rightarrow \partial M \times[0,1$ [, where $p(x)=(x, 0)$ if $x \in \partial M$ and $\mathcal{U}$ is an open subset of $M$ containing $\partial M$. This is the conclusion of Theorem 5.9 in [16] and it will be convenient to summarize the proof. The local retraction theorem (Th. 5.5 in [16]) guarantees the existence of a $\mathcal{C}^{k}$-retraction $r$ from some neighborhood of $\partial M$ onto $\partial M$. On the other hand we can find a $\mathcal{C}^{k}$ function $g$ defined again on some neighborhood of $\partial M$ and such that $g=0$ and the differential $d g$ has rank one on the points of $\partial M$. The restriction of $p=(r, g)$ to some appropriate neighborhood of $\partial M$ is the searched map. From our perspective a crucial point in the above proof is the use of the retraction theorem since this result applies to general submanifolds and does not requires that the submanifold is a boundary. Hence we can adapt the previous proof to our situation $V_{0} \subset W_{0}$ if we select a retraction $r$ from a neighborhood of $V_{0}$ onto $V_{0}$ and a function $g$ defined on a neighborhood of $V_{0}$ and such that $g=0$ on $V_{0}, g>0$ in $M_{0} \backslash V_{0}, g<0$ in $N_{0} \backslash V_{0}$ and the differential $d g$ has rank one everywhere. Since both $r$ and $g$ are of class $\mathcal{C}^{k}$, the same can be said about $p$.

Before the proof of the Smoothing Theorem we need two lemmas. They will be stated now but their proof is postponed to the end of the Appendix.

Lemma 8.1. (a) In the conditions of Theorem 3.2 (a), there exist an open set $\mathcal{V}$ in $W_{0}$ containing $V_{0}$ and a map $\varphi: \mathcal{V} \rightarrow W_{1}$ which coincides with $h$ on $V_{0}$ and is a diffeomorphism from $\mathcal{V}$ onto $\varphi(\mathcal{V})$.

(b) Assume now that the conditions of Theorem $3.2(b)$ hold and let $\mathcal{E}$ be an open subset of $W_{0}$ such that $\operatorname{cl}(\mathcal{E}) \subset \mathcal{G}$. Then the map $\varphi$ can be constructed in such a way that it also coincides with $h$ on $\mathcal{E} \cap \mathcal{V}$.

The next result is a refinement of Lemma 6.1 in [16]. We have changed the notation slightly in order to adapt it to our setting. 
Lemma 8.2. (a) Let $V$ be a manifold without boundary and let $\mathcal{N}$ be an open neighborhood of $V \times\{0\}$ in $V \times \mathbb{R}_{+}$, where $\mathbb{R}_{+}=\left[0, \infty\left[\right.\right.$. Let $g$ be an embedding of $\mathcal{N}$ into $V \times \mathbb{R}_{+}$which equals the identity on $V \times\{0\}$. Then there exists another embedding $\widetilde{g}$ from $\mathcal{N}$ into $V \times \mathbb{R}_{+}$satisfying $\widetilde{g}(\mathcal{N})=g(\mathcal{N}), \widetilde{g}=$ id in a neighborhood of $V \times\{0\}, \widetilde{g}=g$ in a neighborhood of the complement of $\mathcal{N}$.

(b) Assume in addition that $G$ and $E$ are open subsets of $V$ with $\operatorname{cl}(E) \subset G$ and such that $g=$ id on $\left(G \times \mathbb{R}_{+}\right) \cap \mathcal{N}$. Then $\widetilde{g}$ can be constructed with the additional property $\widetilde{g}=\mathrm{id}$ on $\left(E \times \mathbb{R}_{+}\right) \cap \mathcal{N}$.

Proof of Theorem 3.2. (a) In view of Lemma 8.1 we can consider the map $\varphi^{-1} \circ h$, defined in a neighborhood of $V_{0}$ and such that it equals the identity on $V_{0}$. This map can be transported to the cylinder $V_{0} \times \mathbb{R}$ via the map $p: \mathcal{U} \rightarrow V_{0} \times$ ] $-1,1[$ described above. Next we select the domains of the maps so that this process can be made rigorous. The map $\varphi$ is defined on some open set $\mathcal{V}$ and coincides with $h$ on $V_{0}$. Therefore it is possible to find an open set $\mathcal{W}_{*}$ in $W_{0}$ satisfying $V_{0} \subset \mathcal{W}_{*} \subset \mathcal{U} \cap \mathcal{V}$ and $h\left(\mathcal{W}_{*}\right) \subset \varphi(\mathcal{U} \cap \mathcal{V})$. Then the map

$$
\left.g: \mathcal{W} \rightarrow V_{0} \times\right]-1,1\left[, \quad g=p \circ \varphi^{-1} \circ h \circ p^{-1}\right.
$$

with $\mathcal{W}=p\left(\mathcal{W}_{*}\right)$ is a well defined topological embedding.

Next we are going to apply Lemma 8.2 (a) with $V=V_{0}$ and $\mathcal{N}=\mathcal{W} \cap\left(V_{0} \times[0,1[)\right.$. This allows us to construct a diffeomorphism $\tilde{g}: \mathcal{N} \rightarrow g(\mathcal{N})$ and two sets $\mathcal{O}$ and $\mathcal{O}_{*}$ which are open in $V_{0} \times \mathbb{R}_{+}$ and satisfy

$$
\left(V_{0} \times \mathbb{R}_{+}\right) \backslash \mathcal{N} \subset \mathcal{O}, \quad V_{0} \times\{0\} \subset \mathcal{O}_{*} \subset \mathcal{N}, \quad \tilde{g}=g \text { in } \mathcal{O} \cap \mathcal{N}, \quad \tilde{g}=\operatorname{id} \text { in } \mathcal{O}_{*} .
$$

Next we define

$$
f_{+}: M_{0} \rightarrow M_{1}, \quad f_{+}= \begin{cases}\varphi \circ p^{-1} \circ \widetilde{g} \circ p & \text { in } p^{-1}(\mathcal{N}) \\ h & \text { in } M_{0} \backslash p^{-1}(\mathcal{N})\end{cases}
$$

and observe that $f_{+}$and $h$ coincide on $p^{-1}(\mathcal{O} \cap \mathcal{N})$. This shows that $f_{+}$is smooth and we deduce that $f_{+}$has an inverse which is also smooth. Finally we observe that $f_{+}$and $\varphi$ coincide on $p^{-1}\left(\mathcal{O}_{*}\right)$. In this way we have constructed a diffeomorphism between $M_{0}$ and $M_{1}$ that coincides with $\varphi$ in a neighborhood of the boundary of $M_{0}$ and coincides with $h$ far from this boundary. The same process can be applied to construct $f_{-}: N_{0} \rightarrow N_{1}$ and the map

$$
f= \begin{cases}f_{+} & \text {on } M_{0} \\ f_{-} & \text {on } N_{0}\end{cases}
$$

satisfies all the required conditions. This end the proof of part (a).

(b) We start the proof of the second part of the theorem with a remark concerning the notion of clean crossing. The cylinder $\left.V_{0} \times\right]-1,1[$ was employed in the formal definition but it is clear that the interval ] - 1, 1[ plays no essential role. It can be replaced by any interval of the type ] $-\delta, \delta[$ with $\delta>0$. Later we will be interested in reducing the size of the domain of the diffeomorphism $\left.p: \mathcal{U} \rightarrow V_{0} \times\right]-1,1[$. To do this we will consider $\delta \in] 0,1\left[\right.$ and $\mathcal{U}_{\delta}=p^{-1}\left(V_{0} \times\right]-\delta, \delta[)$. The restricted diffeomorphism $\left.p: \mathcal{U}_{\delta} \rightarrow V_{0} \times\right]-\delta, \delta$ [ is admissible for the definition of clean crossing, as it enjoys the properties

$$
p(x)=(x, 0) \text { if } x \in V_{0}, \mathcal{U}_{\delta} \cap \mathcal{E}=p^{-1}(E \times]-\delta, \delta[) .
$$


To prove (b) we will introduce some changes in the construction of $f$ so that it coincides with $h$ on $\mathcal{E}$. The first step is the construction of a subset $G$ of $V_{0}$ with the following properties: $G$ is open in the relative topology of $V_{0}$, the closure of $G$ is compact, $\operatorname{cl}(E) \subset G, p^{-1}\left(\operatorname{cl}(G) \times\left[-\frac{1}{2}, \frac{1}{2}\right]\right) \subset \mathcal{G}$. Then we define the set $\mathcal{G}_{*}=p^{-1}(G \times]-\frac{1}{2}, \frac{1}{2}[)$ and observe that it is an open subset of $W_{0}$ having a clean crossing with $V_{0}$. Since the closure of $\mathcal{G}_{*}$ is contained in $\mathcal{G}$, we can apply Lemma 8.1 (b) to find $\varphi: \mathcal{V} \rightarrow W_{1}$ with $\varphi=h$ on $\mathcal{G}_{*} \cap \mathcal{V}$. In principle this open set $\mathcal{V}$ is unrelated to the set $\mathcal{U}$ appearing in the condition of clean crossing. After constructing the set $\mathcal{W}_{*}$ in the same way as in (a) we find some $\delta<\frac{1}{2}$ such that the set $\mathcal{U}_{\delta} \cap \mathcal{G}_{*}$ is contained in $\mathcal{W}_{*}$. The existence of $\delta$ must be justified. To this end we observe that the compact set $\operatorname{cl}(G) \times[-\delta, \delta]$ converges to $\operatorname{cl}(G) \times\{0\}$ as $\delta$ goes to zero. This convergence is understood in the space of compact subsets of $\left.V_{0} \times\right]-1,1$ [, endowed with the Hausdorff topology. Then $p^{-1}(\operatorname{cl}(G) \times[-\delta, \delta])$ converges to $p^{-1}(\operatorname{cl}(G) \times\{0\})=\operatorname{cl}(G)$, now in the space of compact subsets of $\mathcal{U}$. Since $\operatorname{cl}(G)$ is contained in the open set $\mathcal{W}_{*}$, the same will hold for $p^{-1}(\operatorname{cl}(G) \times[-\delta, \delta])$ when $\delta$ is small. In particular $\mathcal{U}_{\delta} \cap \mathcal{G}_{*}=p^{-1}(G \times]-\delta, \delta[) \subset \mathcal{W}_{*}$.

We can now define the embedding

$$
\left.g: \mathcal{W} \rightarrow V_{0} \times\right]-1,1\left[, \quad g=p \circ \varphi^{-1} \circ h \circ p^{-1}\right.
$$

with $\mathcal{W}=p\left(\mathcal{U}_{\delta} \cap \mathcal{W}_{*}\right)$. Note that the set $\mathcal{W}$ is smaller than the corresponding set in (a). Since $\varphi=h$ on $\mathcal{G}_{*} \cap \mathcal{U}_{\delta} \cap \mathcal{W}_{*}=\mathcal{G}_{*} \cap \mathcal{U}_{\delta}=p^{-1}(G \times]-\delta, \delta[)$, we deduce that $g$ equals the identity on $G \times]-\delta, \delta[$. We can now apply Lemma $8.2($ b) to modify $g$ and obtain $\widetilde{g}: \mathcal{W} \rightarrow g(\mathcal{W})$ satisfying the additional property $\widetilde{g}=$ id on $\left(E \times \mathbb{R}_{+}\right) \cap \mathcal{N}$ where $\mathcal{N}=\mathcal{W} \cap\left(V_{0} \times[0, \delta[)\right.$. The rest of the proof is as in (a)

Proof of Lemma 8.1. (a) Following previous remarks we will construct special neighborhoods of $V_{i}$ in $W_{i}$. Indeed we described the construction for the manifold $W_{0}$ but the same applies to $W_{1}$. For $i=0,1$ we construct diffeomorphisms $\left.p_{i}: \mathcal{U}_{i} \rightarrow V_{i} \times\right]-1,1[$ with

$$
p_{i}(x)=(x, 0) \text { if } x \in V_{i}, \quad p_{i}\left(M_{i} \cap \mathcal{U}_{i}\right)=V_{i} \times\left[0,1\left[, \quad p_{i}\left(N_{i} \cap \mathcal{U}_{i}\right)=V_{i} \times\right]-1,0\right] .
$$

The domains $\mathcal{U}_{i} \subset W_{i}$ are open neighborhoods of $V_{i}$. The restriction of $h$ as a map from $V_{0}$ to $V_{1}$ is a diffeomorphism and the same can be said for

$$
\left.H: V_{0} \times\right]-1,1\left[\rightarrow V_{1} \times\right]-1,1[, \quad H(x, t)=(h(x), t) .
$$

The searched map is $\varphi=p_{1}^{-1} \circ H \circ p_{0}$ defined on $\mathcal{V}=\mathcal{U}_{0}$.

(b) Since $\mathcal{E}$ has a clean crossing with $V_{0}$ we can assume that $\mathcal{E} \cap \mathcal{U}_{0}=p_{0}^{-1}(E \times]-1,1[)$ for some open and relatively compact set $E$ in $V_{0}$. Then we can select two sets $F_{1}$ and $F_{2}$ contained in $V_{0}$, open in the relative topology and having the following properties: $\operatorname{cl}(E) \subset F_{2}, \operatorname{cl}\left(F_{2}\right) \subset F_{1}, \operatorname{cl}\left(F_{1}\right)$ is compact and $p_{0}^{-1}\left(\operatorname{cl}\left(F_{1}\right) \times\left[-\frac{1}{2}, \frac{1}{2}\right]\right) \subset \mathcal{G}$. These sets will be employed later.

In the notations of (a) let $\mathcal{U}_{0}^{*} \subset W_{0}$ be an open set satisfying $V_{0} \subset \mathcal{U}_{0}^{*} \subset \mathcal{U}_{0}$ and $h\left(\mathcal{U}_{0}^{*}\right) \subset \mathcal{U}_{1}$. This is possible because $h$ maps $V_{0}$ onto $V_{1}$. After restricting the size we can assume that $\mathcal{U}_{0}^{*}$ has the following geometric property, $\mathcal{U}_{0}^{*}=p_{0}^{-1}\left(\mathcal{U}^{*}\right)$ where

$$
\mathcal{U}^{*}=\left\{(x, t) \in V_{0} \times\right]-1,1[:|t|<\mu(x)\}
$$

for some continuous function $\left.\mu: V_{0} \rightarrow\right] 0, \frac{1}{2}[$. Next we define the topological embedding

$$
\left.\hat{h}: \mathcal{U}^{*} \rightarrow V_{1} \times\right]-1,1\left[, \quad \hat{h}=p_{1} \circ h \circ p_{0}^{-1} .\right.
$$


This map can be expressed in coordinates

$$
\hat{h}(x, t)=(X(x, t), T(x, t)), \quad(x, t) \in \mathcal{U}^{*} .
$$

In particular, $T(x, 0)=0$ and $X(x, 0)=h(x)$. The last ingredient for our construction will be a $C^{k}$ function $\left.\chi: V_{0} \times\right]-1,1[\rightarrow[0,1]$ satisfying $\chi=1$ in $E \times]-1,1\left[\right.$ and $\chi=0$ in $\left.\left(V_{0} \backslash F_{2}\right) \times\right]-1,1[$. This function can be constructed via a $C^{k}$ partition of unity (see Problem 2.6 in [16]). We are ready to define the map $H: \mathcal{U}^{*} \rightarrow V_{1} \times$ ] $-1,1[$ which will be different from the map defined in (a). Namely,

$$
H(x, t)=(X(x, \chi(x, t) t), \chi(x, t) T(x, t)+(1-\chi(x, t)) t) .
$$

The geometric condition on $\mathcal{U}^{*}$ was imposed to guarantee that $H$ is a well defined map. Moreover $H$ coincides with $\hat{h}$ on $V_{0} \times\{0\}$ and also on $p_{0}\left(\mathcal{E} \cap \mathcal{U}_{0}^{*}\right)=(E \times]-\frac{1}{2}, \frac{1}{2}[) \cap \mathcal{U}^{*}$. We claim that, in contrast to $\hat{h}$, the map $H$ is of class $C^{k}$ in $\mathcal{U}^{*}$. Actually we will prove that there exist two open sets $\mathcal{O}_{1}, \mathcal{O}_{2} \subset \mathcal{U}^{*}$ with $\mathcal{O}_{1} \cup \mathcal{O}_{2}=\mathcal{U}^{*}$ and such that the restriction $H_{\mid \mathcal{O}_{i}}$ is in $C^{k}$ for $i=1,2$. Define

$$
\mathcal{O}_{1}=\left(F_{1} \times\right]-\frac{1}{2}, \frac{1}{2}[) \cap \mathcal{U}^{*}, \quad \mathcal{O}_{2}=\mathcal{U}^{*} \backslash\left(\operatorname{cl}\left(F_{2}\right) \times\right]-\frac{1}{2}, \frac{1}{2}[)
$$

By assumption $\hat{h}$ is smooth on $\mathcal{O}_{1}$. Moreover $\mathcal{O}_{1}$ has the following geometric property: if $(x, t)$ lies in $\mathcal{O}_{1}$ then the same holds for $(x, s)$ if $|s| \leq|t|$. These two properties together with the definition of $H$ imply the smoothness of $H$ on $\mathcal{O}_{1}$. The function $\chi$ vanishes on $\mathcal{O}_{2}$, leading to the identity $H(x, t)=(h(x), t)$ if $(x, t) \in \mathcal{O}_{2}$. The restriction of $h$ to $V_{0}$ is smooth and so the same can be said about the restriction of $H$ to $\mathcal{O}_{2}$.

Once we know that $H$ is $C^{k}$, we are going to apply an inversion result stated in Section 3, namely Corollary 3.4. First of all we observe that $H_{\mid V_{0}}$ is a homeomorphism between $V_{0} \times\{0\}$ and $V_{1} \times\{0\}$. Next we must prove that $\operatorname{det}(d(H)(x, 0)) \neq 0$ for each $x \in V_{0}$. We distinguish two cases: i) Assume $x \in V_{0} \cap \mathcal{O}_{1}$. We know that $\hat{h}_{\mid \mathcal{O}_{1}}$ is an embedding and so $d(\hat{h})(x, 0)$ is a linear isomorphism. From $T(x, 0)=0$ we deduce that $\frac{\partial T}{\partial x}(x, 0)=0$ and $d(\hat{h})(x, 0)$ has a triangular structure. We deduce that

$$
\operatorname{det}(d(\hat{h})(x, 0))=\operatorname{det}\left(\frac{\partial X}{\partial x}(x, 0)\right) \frac{\partial T}{\partial t}(x, 0) \neq 0 .
$$

The manifold $M_{0}$ is mapped by $h$ onto $M_{1}$ and this implies $T(x, t) \geq 0$ whenever $t \geq 0$. The previous observations imply that $\frac{\partial T}{\partial t}(x, 0)>0$. After differentiating $H$ some straightforward computations lead to

$$
\operatorname{det}(d(H)(x, 0))=\left[\begin{array}{c|c}
\frac{\partial X}{\partial x}(x, 0) & \star \\
\hline 0 & \gamma(x)
\end{array}\right]
$$

with $\gamma(x)=\chi(x, 0) \frac{\partial T}{\partial t}(x, 0)+1-\chi(x, 0)$. The quantity $\gamma(x)$ is positive and so the above determinant does not vanish. ii) Assume $x \in V_{0} \cap \mathcal{O}_{2}$. This case is easy because we know that $H(x, t)=(h(x), t)$ and $h$ defines a diffeomorphism between $V_{0}$ and $V_{1}$.

After having checked the assumptions of Corollary 3.4 we can say that there exists an open set $\mathcal{V}^{*} \subset \mathcal{U}^{*}$ with $V_{0} \times\{0\} \subset \mathcal{V}^{*}$ and such that $H$ is a diffeomorphism between $\mathcal{V}^{*}$ and $H\left(\mathcal{V}^{*}\right)$. The map $\varphi=p_{1}^{-1} \circ H \circ p_{0}$ is defined on $\mathcal{V}=p_{0}^{-1}\left(\mathcal{V}^{*}\right)$ and satisfies all the required properties. 
Proof of Lemma 8.2. For the proof of (a) we refer to Lemma 6.1 in Munkres' monograph [16]. To prove (b) we need to modify some of the arguments in [16]. First of all we observe that it is not restrictive to assume that $\mathcal{N}$ has the special form

$$
\mathcal{N}_{\beta}=\left\{(x, t) \in V \times \mathbb{R}_{+}: 0 \leq t<\beta(x)\right\}
$$

where $\beta: V \rightarrow] 0,1\left[\right.$ is a $C^{k}$ function. This follows from the condition $g=\widetilde{g}$ in the neighborhood of the complement of $\mathcal{N}$.

After expressing $g$ in coordinates, $g=(X, T)$, we notice that $T(x, 0)=0$ and, since $g$ is an embedding, we conclude that $\frac{\partial T}{\partial t}(x, t)>0$ for every $x \in V$. We claim that there exist two $C^{k}$ functions $\epsilon: V \rightarrow] 0,1]$ and $\left.\beta_{1}: V \rightarrow\right] 0,1\left[\right.$ such that $\beta_{1}<\beta$ and

$$
\epsilon(x)=1 \text { if } x \in E \text { and } \frac{\partial T}{\partial t}(x, t) \geq \epsilon(x) \text { if } x \in V, t<\beta_{1}(x) .
$$

To construct $\beta_{1}$ we recall the positivity of $\frac{\partial T}{\partial t}$ on $t=0$ and find a neighborhood of $V \times\{0\}$ where $\frac{\partial T}{\partial t}$ is still positive. Then we can find $\beta_{1}$ such that the closure of $\mathcal{N}_{\beta_{1}}$ is contained in this neighborhood. That is, $\frac{\partial T}{\partial t}(x, t)>0$ if $x \in V, 0 \leq t \leq \beta_{1}(x)$. The construction of $\epsilon$ is more delicate. First we define

$$
\nu: V \rightarrow] 0,1], \quad \nu(x)=\min \left[1, \min \left\{\frac{\partial T}{\partial t}(x, t): 0 \leq t \leq \beta_{1}(x)\right\}\right] .
$$

Next we construct a covering of $V$ by open sets $U_{i}^{*}$ having a compact closure and such that the following property holds: if $U_{i}^{*} \cap E \neq \emptyset$ then $\operatorname{cl}\left(U_{i}^{*}\right) \subset G$. A locally finite covering $\left\{U_{i}\right\}$ of open sets with $U_{i} \subset U_{i}^{*}$ can be constructed together with a $C^{k}$ partition of unity $\left\{\varphi_{i}\right\}$ dominated by $\left\{U_{i}\right\}$; that is, $0 \leq \varphi_{i} \leq 1, \sum_{i} \varphi_{i}=1$ everywhere and $\operatorname{supp}\left(\varphi_{i}\right) \subset U_{i}$. Define $m_{i}=\min \left\{\nu(x): x \in \operatorname{cl}\left(U_{i}\right)\right\}$ and $\epsilon(x)=\sum_{i} m_{i} \varphi_{i}(x)$. Let us check that this function has the required properties. Given $x \in E$, if $i$ is an index with $\varphi_{i}(x)>0$ then $\operatorname{supp}\left(\varphi_{i}\right) \cap E$ is non-empty. This implies that also $U_{i}^{*} \cap E$ is non-empty and therefore $U_{i} \subset U_{i}^{*} \subset G$ and $m_{i}=1$. This argument works for all points $x \in E$ and all indexes with $\varphi_{i}(x)>0$ so that $\epsilon(x)=\sum_{i} \varphi_{i}(x)=1$. Given any $x \in V, \nu(x) \geq m_{i}$ whenever $\varphi_{i}(x)>0$. This implies $\epsilon(x) \leq \nu(x)$ and so $\epsilon(x) \leq \frac{\partial T}{\partial t}(x, t)$ if $t \leq \beta_{1}(x)$.

The rest of the proof will consist in a sequence of modifications of the original map $g$ leading to the searched map $\widetilde{g}$. All intermediate maps will be denoted by $g_{i}: \mathcal{N}_{\beta} \rightarrow V \times \mathbb{R}_{+}, i=1,2, \ldots$ with coordinates $g_{i}=\left(X_{i}, T_{i}\right)$ and satisfying the properties: $g_{i}$ is an embedding and $g_{i}\left(\mathcal{N}_{\beta}\right)=g\left(\mathcal{N}_{\beta}\right)$, $g_{i}=$ id on $V \times\{0\}$ and also on $\left(E \times \mathbb{R}_{+}\right) \cap \mathcal{N}_{\beta}, g_{i}=g_{i-1}$ in a neighborhood of $\left(V \times \mathbb{R}_{+}\right) \backslash \mathcal{N}_{\beta}$. We use the convention $g_{0}=g$ and sum up the above properties by saying that $g_{i}$ is in the class $\mathcal{M}$. The rest of the proof is organized in three steps.

Step 1. There exists $g_{1} \in \mathcal{M}$ satisfying $\frac{\partial T_{1}}{\partial t}(x, t) \geq 1$ in a neighborhood of $V \times\{0\}$.

Let $\alpha \in C^{\infty}(\mathbb{R})$ be a monotone function satisfying $\alpha(t)=0$ if $t \leq \frac{1}{3}$ and $\alpha(t)=1$ if $t \geq \frac{2}{3}$. As in [16] we define

$$
\Psi(x, t)=\left(1-\alpha\left(\frac{t}{\beta_{1}(x)}\right)\right) \epsilon(x) t+\alpha\left(\frac{t}{\beta_{1}(x)}\right) t
$$

and observe that $\Psi$ is $C^{k}$ and $\Psi(x, \cdot)$ is an increasing diffeomorphism of the interval $\left[0, \beta_{1}(x)\right]$. Indeed, $\Psi(x, 0)=0, \Psi\left(x, \beta_{1}(x)\right)=\beta_{1}(x)$ and $\frac{\partial \Psi}{\partial t}=(1-\alpha) \epsilon+\alpha+\frac{\alpha^{\prime}}{\beta_{1}}(1-\epsilon) t>0$. The map $\eta_{1}: \operatorname{cl}\left(\mathcal{N}_{\beta_{1}}\right) \rightarrow \operatorname{cl}\left(\mathcal{N}_{\beta_{1}}\right), \eta_{1}(x, t)=(x, \Psi(x, t))$ is a diffeomorphism and we define

$$
g_{1}: \mathcal{N}_{\beta} \rightarrow V \times \mathbb{R}_{+}, \quad g_{1}= \begin{cases}\eta_{1}^{-1} \circ g & \text { in } \mathcal{N}_{\beta_{1}} \\ g & \text { otherwise } .\end{cases}
$$


Since $\eta_{1}$ equals the identity for $t \geq \frac{2}{3} \beta_{1}(x)$, this map is an embedding with $g_{1}\left(\mathcal{N}_{\beta}\right)=g\left(\mathcal{N}_{\beta}\right)$. Moreover the condition $\epsilon=1$ on $E$ implies that $\eta_{1}$ also equals the identity on $\left(E \times \mathbb{R}_{+}\right) \cap \mathcal{N}_{\beta}$. It is now clear that $g_{1}$ belongs to the class $\mathcal{M}$. To check the additional condition imposed on $\frac{\partial T_{1}}{\partial t}$ we observe that $\eta_{1}^{-1}(x, t)=\left(x, \frac{t}{\epsilon(x)}\right)$ if $t \leq \frac{\epsilon(x) \beta_{1}(x)}{3}$. Let $\left.\beta_{2}: V \rightarrow\right] 0,1\left[\right.$ be a $C^{k}$ function such that $T(x, t) \leq \frac{\epsilon(x) \beta_{1}(x)}{3}$ if $0 \leq t \leq \beta_{2}(x)$. Then if $(x, t)$ lies in $\mathcal{N}_{\beta_{2}}, \frac{\partial T_{1}}{\partial t}(x, t)=\frac{1}{\epsilon(x)} \frac{\partial T}{\partial t}(x, t) \geq 1$.

Step 2. There exists $g_{2} \in \mathcal{M}$ satisfying $T_{2}(x, t)=t$ in a neighborhood of $V \times\{0\}$.

Given $(x, t) \in \mathcal{N}_{\beta_{2}}$, consider the equation with unknown $\tau$,

$$
F(x, t ; \tau):=\left(1-\alpha\left(\frac{t}{\beta_{2}(x)}\right)\right) T_{1}(x, \tau)+\alpha\left(\frac{t}{\beta_{2}(x)}\right) \tau-t=0 .
$$

Since $\frac{\partial T_{1}}{\partial t}(x, t) \geq 1$ if $0 \leq t \leq \beta_{2}(x)$ we deduce that $T_{1}(x, t) \geq t$. This inequality implies that $F(x, t ; \cdot)$ has a change of sign, $F(x, t ; 0) \leq 0 \leq F\left(t, x ; \beta_{2}(x)\right)$, and so $F=0$ has at least one solution $\tau$ in $\left[0, \beta_{2}(x)\right]$. This solution is unique because $F(t, x ; \cdot)$ is strictly increasing. Actually, $\frac{\partial F}{\partial \tau}=(1-\alpha) \frac{\partial T_{1}}{\partial t}+\alpha \geq 1$. The positivity of this derivative also implies that $\tau=\tau(x, t)$ is of class $C^{k}$ on $x \in V, 0 \leq t \leq \beta_{2}(x)$. For future use we note that $T_{1}(x, \tau(x, t))=t$ if $0 \leq t \leq \frac{1}{3} \beta_{2}(x)$ and $\tau(x, t)=t$ if $t \geq \frac{2}{3} \beta_{2}(x)$. In particular, $\tau(x, 0)=0$ and $\tau\left(x, \beta_{2}(x)\right)=\beta_{2}(x)$. By implicit differentiation,

$$
\left[(1-\alpha) \frac{\partial T_{1}}{\partial t}+\alpha\right] \frac{\partial \tau}{\partial t}=1+\frac{\alpha^{\prime}}{\beta_{2}}\left(T_{1}-\tau\right) \geq 1
$$

implying that $\frac{\partial \tau}{\partial t}(x, t)>0$. The map $\eta_{2}(x, t)=(x, \tau(x, t))$ is a diffeomorphism of the closure of $\mathcal{N}_{\beta_{2}}$. It equals the identity on $t \geq \frac{2}{3} \beta_{2}(x)$ and also on $t=0$. Since $T_{1}(x, t)=t$ if $x \in E$, $0 \leq t \leq \beta_{2}(x)$, we deduce that $\tau(x, t)=t$ if $x \in E$ and so $\eta_{2}$ is also the identity on $\left(E \times \mathbb{R}_{+}\right) \cap \mathcal{N}_{\beta_{2}}$. The map

$$
g_{2}: \mathcal{N}_{\beta} \rightarrow V \times \mathbb{R}_{+}, \quad g_{2}= \begin{cases}g_{1} \circ \eta_{2} & \text { in } \mathcal{N}_{\beta_{2}} \\ g & \text { otherwise }\end{cases}
$$

is in $\mathcal{M}$ and satisfies $T_{2}(x, t)=T_{1}(x, \tau(x, t))=t$ if $0 \leq t \leq \frac{1}{3} \beta_{2}(x)$.

Step 3. In this step we proceed exactly as in [16]. We sketch the main ideas of the construction and refer to [16] for more details. As in [16] we employ the notation $Y=\mathcal{N}_{\beta_{2}}$. The manifold $V$ can be covered by a locally finite collection $\left\{U_{i}\right\}_{i \in I}$ of open subsets of $V$ such that the closure of each $U_{i}$ is diffeomorphic to a closed ball in $\mathbb{R}^{n-1}$. The set of indexes can be chosen at most countable and we will assume that it is either a finite set of the type $I=\{i \in \mathbb{N}: 3 \leq i \leq N\}$ or the infinite set $I=\{3,4,5, \ldots\}$. We select a second covering of $V$, now by compact sets $C_{i} \subset U_{i}$. Finally $V_{i}$ will be open sets (in $V$ ) sastisfying $C_{i} \subset V_{i}$ and $\operatorname{cl}\left(V_{i}\right) \subset U_{i}$. Let $c_{3}>0$ be such that $U_{3} \times\left[0, c_{3}\right]$ is contained in $Y$ and consider the restriction of $g_{2}$ to this set. We apply Lemma 6.2 in [16] to find an embedding $g_{3}: U_{3} \times\left[0, c_{3}\right] \rightarrow V \times \mathbb{R}_{+}$satisfying $g_{3}\left(U_{3} \times\left[0, c_{3}\right]\right)=g_{2}\left(U_{3} \times\left[0, c_{3}\right]\right), T_{3}=t$ and the properties below,

(1) $g_{3}=$ id on $U_{3} \times\{0\}$ and on $C_{3} \times\left[0, \delta_{3}\right]$ for some $\delta_{3}>0$

(2) $X_{3}=X_{2}$ outside $V_{3} \times\left[0, \frac{c_{3}}{2}\right]$

(3) If $g_{2}$ is the identity on $\{x\} \times[0, b]$ for some $x \in U_{3}$ and $b<\beta_{2}(x)$, then $g_{3}$ is also the identity on this set.

This last property is important because it guarantees that $g_{3}=\mathrm{id}$ on $\left(E \times \mathbb{R}_{+}\right) \cap Y$. The map $g_{3}$ can be extended to $Y$ via the formula $g_{3}=g_{2}$ on $Y \backslash\left(U_{3} \times\left[0, c_{3}\right]\right)$. The embedding $g_{3}: Y \rightarrow g_{2}(Y)$ 
satisfies $T_{3}=t$ everywhere and $g_{3}=\mathrm{id}$ on $C_{3} \times\left[0, \delta_{3}\right]$ for some $\delta_{3}<\frac{\beta_{2}(x)}{2}$. The process can be repeated inductively on the set $I$ and the figures below illustrate the passage from $g_{i-1}$ to $g_{i}$. The horizontal axis is the manifold $V$ and the regions in blue are $C_{i} \times\left[0, \delta_{i}\right]$. The map is not modified in the white region.
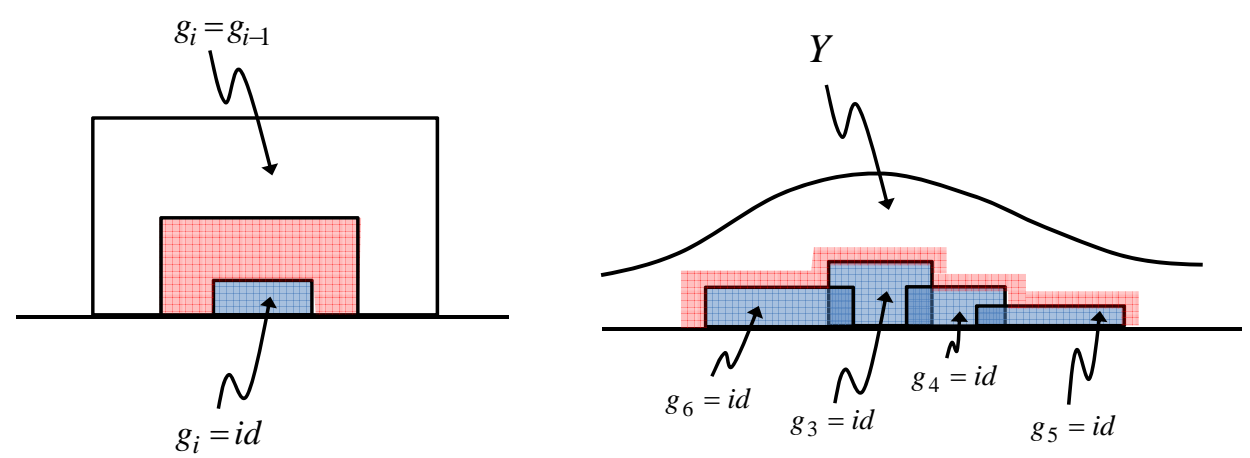

Figure 5: Construction of $g_{i}$ and overlapping of the succesive modifications.

The succesive modifications preserve the previous conditions $g_{i-1}=\mathrm{id}$ on $C_{j} \times\left[0, \delta_{j}\right], j<i$, thanks to property (3). The same can be said about the condition $g_{i-1}=\mathrm{id}$ on $\left(E \times \mathbb{R}_{+}\right) \cap Y$. If the set $I$ is finite we find, after a finite number of modifications, the map

$$
\widetilde{g}: \mathcal{N}_{\beta} \rightarrow V \times \mathbb{R}_{+}, \quad \widetilde{g}= \begin{cases}g_{N} & \text { in } Y \\ g_{2} & \text { otherwise }\end{cases}
$$

If $I$ is infinite then $\widetilde{g}$ is defined by

$$
\widetilde{g}(x, t)=\lim _{i \rightarrow \infty} g_{i}(x, t) \quad \text { if }(x, t) \in Y .
$$

Then $\widetilde{g}$ is extended to $\mathcal{N}_{\beta}$ by letting $\widetilde{g}=g_{2}$ on $\mathcal{N}_{\beta} \backslash Y$.

\section{Acknowledgments}

The first and second authors are supported by a MINECO grant number MTM2013-40998-P. The third author by a MINECO grant number MTM2014-52209-C2-1-P and the fourth author by a MINECO/FEDER grant number MTM2014-52232-P. The first two authors are also supported by a CIRIT grant number 2014SGR568.

\section{References}

[1] R.H. Bing. A homeomorphism between the 3-sphere and the sum of two solid horned spheres, Ann. of Math. 56 (1952), 354-362. 
[2] R. H. Bing. Inequivalent families of periodic homeomorphisms of $E^{3}$, Ann. of Math. 80 (1964), 78-93.

[3] A. Cima, A. Gasull, F. Mañosas. Simple examples of planar involutions with non-global Montgomery-Bochner linearizations, Appl. Math. Lett. 25 (2012) 2086-2088.

[4] A. Cima, A. Gasull, F. Mañosas and R. Ortega Linearization of planar involutions in $\mathcal{C}^{1}$, to appear in Ann. Mat. Pur. Appl.

[5] P. E. Conner and E. E. Floyd. On the construction of periodic maps without fixed points, Proc. Amer. Math. Soc. 10 (1959), 354-360.

[6] A. Constantin and B. Kolev. The theorem of Kerékjártó on periodic homeomorphisms of the disc and the sphere, Enseign. Math. 40 (1994), 193-204.

[7] D. B. A. Epstein, Pointwise periodic homeomorphisms, Proc. London Math. Soc. 42 (1981), 415-460.

[8] R. Haynes, S. Kwasik, J. Mast and R. Schultz. Periodic maps on $\mathbf{R}^{7}$ without fixed points, Math. Proc. Cambridge Philos. Soc. 132 (2002), 131-136.

[9] M. W. Hirsch. "Differential Topology", Springer-Verlag, New York 1976.

[10] J. M. Kister. Differentiable periodic actions on $E^{8}$ without fixed points, Amer. J. Math. 85 (1963), 316-319.

[11] M. Kuczma, B. Choczewski and R. Ger. "Iterative functional equations". Encyclopedia of Mathematics and its Applications 32. Cambridge University Press, Cambridge, 1990.

[12] K.R. Meyer, G.R. Hall, D. Offin. "Introduction to Hamiltonian Dynamical Systems and the $N$-Body Problem", Springer, 2009.

[13] D. Montgomery, Pointwise Periodic Homeomorphisms, Amer. J. Math. 59 (1937), 118-120.

[14] D. Montgomery and L. Zippin. "Topological transformation groups", Interscience, New York 1955.

[15] M. Morse. Differentiable mappings in the Shoenflies theorem, Compositio Math. 14 (1959), 83-151.

[16] J. R. Munkres. "Elementary Differential Topology", Princeton University Press, Princeton 1966

[17] R. Ortega. Retracts, fixed points and differential equations, Rev. R. Acad. Cien. Serie A. Mat. 102 (2008) 89-100.

[18] R. Ortega. A dynamical characterization of planar symmetries, Qual. Theory Dyn. Syst. 10 (2011) 197-201. 